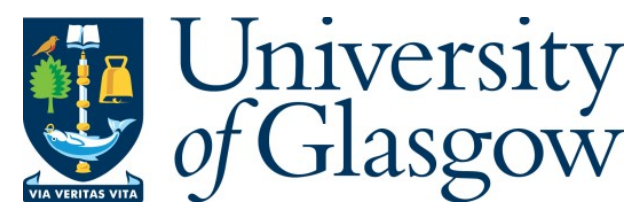

El Hamdaoui, M., Merodio, J. and Ogden, R.W. (2020) Two-phase piecewise homogeneous plane deformations of a fibre-reinforced neo-Hookean material with application to fibre kinking and splitting. Journal of the Mechanics and Physics of Solids, 143, 104091.

(doi: $10.1016 /$ j.jmps.2020.104091)

This is the Author Accepted Manuscript.

There may be differences between this version and the published version. You are advised to consult the publisher's version if you wish to cite from it.

https://eprints.gla.ac.uk/220295/

Deposited on: 13 July 2020

Enlighten - Research publications by members of the University of Glasgow http://eprints.gla.ac.uk 


\title{
Two-phase piecewise homogeneous plane deformations of a fibre-reinforced neo-Hookean material with application to fibre kinking and splitting
}

\author{
${ }^{1} \mathrm{M}$. El Hamdaoui, ${ }^{2}$ J. Merodio and ${ }^{3} \mathrm{R}$.W Ogden \\ ${ }^{1}$ Department of Ophthalmology, University of Alabama at Birmingham \\ Birmingham, AL 35294, USA \\ ${ }^{2}$ Department of Continuum Mechanics and Structures \\ Universidad Politécnica de Madrid, 28040, Madrid, Spain \\ ${ }^{3}$ School of Mathematics and Statistics, University of Glasgow \\ Glasgow G12 8SQ, UK. Email: raymond.ogden@glasgow.ac.uk
}

\begin{abstract}
Two-phase piecewise homogeneous plane deformations are examined in respect of a neo-Hookean matrix material reinforced with embedded aligned fibres characterized by a single stiffness parameter. The deformations are interpreted in terms of fibre kinking and fibre splitting. Previous work has shown that such a transversely isotropic material can lose ellipticity if the reinforcing stiffness is sufficiently large and the fibre direction is sufficiently compressed. In particular, it was shown that the associated failure modes are characterised by the emergence of weak surfaces of discontinuity that are normal to the fibre direction (the onset of fibre kinking) or parallel to the fibre direction (the onset of fibre splitting). Here, the analysis of strong surfaces of discontinuity, developing from weak ones, is studied. The considered model can give rise to piecewise smooth plane deformations separated by a plane stationary surface of discontinuity, interpreted as either kinking or splitting. Attention is restricted to (plane) deformations in which, on one side of the surface of discontinuity, the load axis is aligned with the fibre axis. Then the fibre stretch on this side of the discontinuity is a natural load parameter. The ellipticity status of the two-phase piecewise homogeneous plane deformations is shown to span all four possible ellipticity/non-ellipticity permutations. If both deformation states are elliptic, then a suitable intermediate deformation is shown to be non-elliptic. Moreover, it is shown that the mechanism is dissipative, and maximally dissipative quasi-static failure motion is examined in respect of both kinking and splitting. It follows that, firstly, surfaces of discontinuity perpendicular to the fibre direction, associated with fibre kinking, are nucleated followed by surfaces of discontinuity parallel to the fibre direction, associated with fibre splitting. With respect to kinking, such maximally dissipative kinks nucleate only in compression as weak surfaces of discontinuity, with the subsequent motion converting non-elliptic deformation to elliptic deformation.
\end{abstract}

Keywords: Discontinuous finite deformations; Fibre-reinforced materials; Fibre kinking; Fibre splitting. 


\section{Introduction}

Based on the implications of the loss of ellipticity of the governing equations of equilibrium for fibre-reinforced incompressible nonlinearly elastic solids, the purpose of the present work is to examine the initiation of kink band instabilities and the possible coincidence of kinking and splitting in such materials. To set the scene we first consider some background on shear band initiation and related instabilities.

\subsection{Background and motivation}

A general theoretical framework for shear bands was provided by Hill (1962), while, with a view to establishing a realistic model capable of predicting the critical bifurcation stress (and/or strain) leading to formation of a shear band, different constitutive models were analysed in a variety of contexts. For example, Rice (1976) showed that some isotropic elastic solids, and elastic-plastic solids with a smooth yield surface, do not develop shear band instabilities, while important theoretical contributions were made by Hill and Hutchinson (1975), Anand and Spitzig (1980) and Hutchinson and Tvergaard (1981) within the theory of plasticity under several loading conditions. In the context of the inelastic behaviour of over-consolidated clay soils shear band formation was investigated by Rice (1973) and Rudnicki and Rice (1975).

In connection with asymptotic studies of crack problems, Knowles and Sternberg (1978, 1980) showed that loss of ellipticity of the field equations of nonlinear compressible hyperelastic materials under plane deformation is a necessary condition for the emergence of solutions lacking the standard smoothness properties required by the governing differential equations of equilibrium. Identification of the surfaces on which ellipticity fails formed the subject of an earlier paper (Knowles and Sternberg, 1976), while Knowles and Sternberg (1978) were concerned with the emergence of surfaces of discontinuity and piecewise homogeneous deformations and energy considerations associated with the loss of ellipticity. and dissipation (see also Abeyaratne, 1980; Abeyaratne and Knowles, 1989; Knowles, 1979).

Budiansky and Fleck (1993) provided one of the most widely used models that incorporates the effect of combined stress loading for predicting realistic ranges of kink band angles in composites. Similar analyses were conducted by Sutcliffe and Fleck (1994) and Moran et al. (1995) for carbon fibre epoxy composites, by Moran and Shih (1998) for ductile matrix fibre composites, by Poulsen et al. (1997) for wood, and for an advanced fibre-reinforced composite by Kyriakides et al. (1995) and Vogler and Kyriakides (1997). These works focus not only on predicting the compressive strength but also on the propagation of the kink band. It has been assumed in the literature that a kink band may start from either a well-defined initial band of wavy fibres (for example, Kyriakides et al., 1995; Kyriakides and Ruff, 1997) or from a deformation induced band, as in, for example, Christoffersen and Jensen (1996), Jensen and Christoffersen (1997) and Christensen and DeTeresa (1997).

Analysis of discontinuous deformation gradients, forming two-phases of the same material, provides both qualitative and quantitative information on the formation and broadening of (shear) kink bands (Merodio and Pence, 2001a b). Two different phases of a given material formed by two joined homogeneously deformed half-spaces have been studied by Fu and Freidin (2004) with respect to (quasi-static) bifurcation and stability using a kinetic stability criterion and an energy analysis. Furthermore, Fu and Zhang (2006) analysed kink band formation with respect to the choice of the strain-energy function and the stability of the solutions based on the so-called Maxwell relation. Considering uni-directionally fibre-reinforced materials, they obtained the kink propagation stress, the kink orientation 
angle and the fibre direction within the kink band. More recently, Baek and Pence (2010) studied the effect of shearing deformation in fibre-reinforced materials on the emergence and disappearance of kink surfaces for several different boundary-value problems.

Experiments by Lee and Anthony (1999) examined the effect of fibre diameter and initial misalignment angle on the compressive behaviour of glass fibre unidirectionally-reinforced composites and the prediction of their compressive strength for a wide range of fibre volume fractions. The initial fibre misalignment angle and the axial propagation of kink bands have also been studied experimentally by Kyriakides et al. (1995) and Kyriakides and Ruff (1997). Lee et al. (2000) observed experimentally that glass fibre epoxy composites fail predominantly by a splitting failure mode at lower fibre volume fractions and by a combination of splitting and kinking at higher fibre volume fractions, while, by contrast, carbon fibre epoxy composites were found to fail only by kinking.

With this background in mind, the motivation for the present work is to provide qualitative understanding of kink band phenomena in fibre-reinforced materials, with special attention to the simultaneous existence of fibre kinking and fibre splitting observed by Prabhakar and Waas (2013a). Various efforts have been aimed at developing models that can capture and predict fibre failure (Yerramalli and Waas, 2004, Prabhakar and Waas, 2013a b; El Hamdaoui et al., 2015, 2018; Hasanyan and Waas, 2018) since, to some extent, the use of fibre-reinforced materials has been constrained by lack of understanding of the failure mechanisms.

To analyze and predict failure mechanisms, micromechanics approaches that consider the geometry of the microstructure and the imperfections associated with them have been used to obtain the load bearing capacity of the material (see Prabhakar and Waas, 2013b and references therein). It is therefore essential to consider models that account for fibre and matrix constituents of the composite since such models provide physical insight into the failure of both the fibre and the matrix, as well as the load transfer between them. However, micromechanics approaches are often less practical than macromechanical models since, for example, they are more costly in computational time due to the large number of degrees-offreedom involved. Our preferred approach is therefore to adopt a macroscopic continuum model that embodies information about the microstructure, such as fibre orientation.

Within a macroscopic continuum framework, initiation of material failure is often associated with the loss in ellipticity of the governing equations. Analysis of ellipticity is therefore important, not only for characterizing macromechanical behaviour and failure but also, for instance, in computational mechanics, where loss of ellipticity leads to meshdependent results that depend on the mesh size, and this needs to be well understood (Hasanyan and Waas, 2018).

A continuum-mechanical model in the setting of nonlinear elasticity theory that predicts the onset of material instabilities for fibre-reinforced materials has been established in a series of paper by Merodio and Ogden (2002, 2003, 2005a b c and references therein. The loss of stability and the onset of fibre failure in fibre-reinforced materials were related to the ellipticity status of the governing equation of equilibrium, a status which changes locally in type as a result of deformation. This change is referred to as loss of (ordinary) ellipticity, which can be interpreted as the onset of a failure mechanism.

For a given strain-energy function the loss of ellipticity condition determines both the deformation associated with the existence of surfaces of weak discontinuity and the direction of the normal to that surface. Surfaces of weak discontinuity (or weak surfaces) are surfaces across which the second derivative of the deformation field is discontinuous. For some historical background related to this type of discontinuity it is of interest to consult the book by Hadamard (1903). It has been found in Merodio and Ogden (2002) 
that for reinforcing models that depend only on the fibre stretch ellipticity is lost under fibre contraction, and the associate failure mode is fibre kinking. On the other hand, for reinforcing models that depend on the shear as well as stretch the failure mechanism can involve combination of fibre kinking and fibre splitting.

These failure modes are characterised by the emergence of weak surfaces of discontinuity that are normal to the fibre direction (fibre kinking) or parallel to the fibre direction (fibre splitting). Weak surfaces do not provide information about either the (quasi-static) development of the discontinuities or the different kinematic and stress variables across the surfaces of discontinuity. These data are provided by surfaces of strong discontinuity. In a fully developed or strong surface of discontinuity the first derivative of the deformation field (i.e. the deformation gradient) suffers a finite jump.

Surfaces of strong discontinuity, also referred as elastostatic shocks, non-evolutionary jump discontinuities, phase boundaries, stationary kink surfaces, stationary kinks, etc., were considered in (Merodio and Pence, 2001a b) for analyzing fibre kinking using a reinforcing model that depends on the fibre stretch. Their analysis focused on the (plane) deformation gradients across the shock, where, in addition, one of the (in-plane) principal stretches associated with one side of the shock was aligned with the fibre direction. Henceforth in this paper, for simplicity of terminology, we mainly refer to surfaces of discontinuity as (elastostatic) shocks, although they are not shocks in the conventional sense.

The kink band is given by a three-zone state with the kink zone separating the two sides of the band (designated the ' + ' and ' - ' sides). However, it should be emphasized that the actual width of a kink band and its development is not captured by the pure elasticity theory used here. This requires a more general constitutive theory such as a second-gradient theory.

Our aim is to exploit the elasticity theoretical framework further in order to capture the combination of fibre kinking and fibre splitting. This requires the emergence of equilibrated shocks that are able to describe kinking and splitting simultaneously to be accommodated. The onset of fibre splitting also has a three-zone state, where in this case there is a splitting zone separating the ' + ' and '-' sides of the discontinuity. It can be argued that fibre splitting is not related to continuous displacements, as it is in fact a catastrophic failure mechanism. However, analysis of piecewise homogeneous deformations provides useful input for understanding and describing the mechanisms involved. Indeed, connecting the fibre stretching and shearing with the formation of shocks, i.e. with the formation of different fibre failure mechanisms, can be exploited in the nonlinear constitutive modelling of fibre-reinforced materials.

\section{$1.2 \quad$ Structure of the paper}

The main goals of the present analysis are to predict the combined splitting-kinking failure mode observed experimentally by Lee et al. (2000) and to capture the evolution of the elastostatic shock direction and kinking angle. Two-phase deformations of a fibre-reinforced material that correspond to two different phases of the same elastic material are constructed and analyzed for this purpose. The material model adopted here consists of a neo-Hookean matrix in which are embedded aligned fibres so that the material response is transversely isotropic. The fibres are characterized by a so-called reinforcing model that penalizes deformation in the fibre direction, and the overall material strain-energy function is taken to be the sum of the neo-Hookean and reinforcing energy functions.

Sections 24 focus on purely mechanical aspects of shocks. In Section 2, the main equations are presented. These include the requirements of displacement continuity and 
traction continuity that have to be satisfied by the set of all considered piecewise homogeneous plane deformations. In Section 3 some special cases are studied, namely both weak and strong shocks orthogonal and parallel to the fibre direction, while more general shocks are analyzed in Section 4. The ellipticity status of the piecewise homogeneous plane deformations is also provided. The results show that the existence of a strong shock involves loss of ellipticity at a suitable intermediate deformation, in agreement with previous analyses.

In Section 5, quasi-static shock motion is considered based on a detailed energy analysis involving the use of the so-called driving traction, i.e. the magnitude of a fictitious nominal traction acting on the shock by the surrounding material. Following (Merodio and Pence, 2001a b) two families of solutions are studied with the purpose of establishing a criterion for selecting shock evolution, both for non-dissipative solutions and maximally dissipative shocks, which can be considered as two extreme cases. For sufficiently small values of the reinforcing parameter, as fibre contraction is increased, non-dissipative shocks nucleate at the particular values that give the first modes associated with loss of ellipticity in, for example, the ' + ' zone. Incipient loss of ellipticity may occur in two different modes, which are associated with fibre kinking and fibre splitting. It follows that, firstly, shocks perpendicular to the fibre direction, associated with fibre kinking, are nucleated followed by shocks parallel to the fibre direction, associated with fibre splitting, and the quasi-static evolution of both kinking and splitting is analyzed.

Non-dissipative motion or neutral stability (analogous to the Gibbs and Maxwell phase equilibrium conditions) from the values of the ' + ' side gives rise to a simultaneous rapid (almost instantaneous) large increase of the fibre contraction together with a rapid escalation of shear deformation, subsequently referred to as 'fibre shearing', in turn associated with an increase in the transverse strain. This occurs at the point of loss of ellipticity for any value of the reinforcing parameter, and, for sufficiently large values of the fibre reinforcement, a snap-back mechanism is associated with the non-dissipative kinking solutions. This means that the considered failure mechanisms are clearly dissipative, i.e. energy is dissipated in the process of kink band and splitting formation (see Prabhakar and Waas, 2013b). It has been noted that large strains within the kink band suggest fibre/matrix splitting, which has been observed in experiments in conjunction with the formation of kink banding for certain materials (Prabhakar and Waas, 2013a).

Maximally dissipative shocks are also studied. Then no snap-back is found and a unique solution is singled out in the '-' zone for both kinking and splitting, as for the nondissipative case, although in that case just for sufficiently small values of the reinforcing parameter. Therefore, two families (kinking and splitting) nucleate, each being associated with the two different failure modes given by the breakdown of ellipticity in the ' + ' zone. During subsequent motions for the maximally dissipative kinking solutions, there is a rapid increase of fibre contraction and fibre shearing while non-elliptic deformations convert into elliptic deformations. With respect to fibre kinking angles, as well as kink orientation angles, it is shown that maximally dissipative solutions might capture experimental observations closely although we are dealing here with just a simple prototype model and our results have a qualitative rather than a quantitative meaning. In addition, with respect to fibre splitting, maximally dissipative shocks are associated with fibre angles in the splitting zone which are almost zero and there is also a rapid increase of fibre contraction and fibre shearing. 


\section{Basic Equations}

\subsection{Material model under plane strain}

We consider an incompressible elastic material under plane strain deformation. Let $\mathcal{D}$ be the (plane) domain occupied by a body in its undeformed configuration and $\mathcal{D}^{*}$ be the (plane) domain occupied by the same body in its deformed configuration. Vectors and tensors are described with respect to the planar orthonormal bases $\left\{\mathbf{E}_{i}\right\}, i=1,2$, in $\mathcal{D}$, and $\left\{\mathbf{e}_{i}\right\}, i=1,2$, in $\mathcal{D}^{*}$. Let $\boldsymbol{\chi}$ denote the smooth invertible mapping that deforms $\mathcal{D}$ onto $\mathcal{D}^{*}$ according to

$$
\mathbf{x}=\chi(\mathbf{X})=\mathbf{X}+\mathbf{u}(\mathbf{X})
$$

where $\mathbf{X}=X_{1} \mathbf{E}_{1}+X_{2} \mathbf{E}_{2}$ and $\mathbf{x}=x_{1} \mathbf{e}_{1}+x_{2} \mathbf{e}_{2}$ are the position vectors of a material point in the undeformed and deformed configurations, respectively, while $\mathbf{u}=u_{1}\left(X_{1}, X_{2}\right) \mathbf{E}_{1}+$ $u_{2}\left(X_{1}, X_{2}\right) \mathbf{E}_{2}$ is the corresponding displacement vector. The (two-dimensional) deformation gradient tensor is $\mathbf{F}=\partial \boldsymbol{\chi} / \partial \mathbf{X}$, and the associated right and left Cauchy-Green deformation tensors are $\mathbf{C}=\mathbf{F}^{\mathrm{T}} \mathbf{F}$ and $\mathbf{B}=\mathbf{F F}^{\mathrm{T}}$, respectively. The incompressibility constraint given by $\operatorname{det} \mathbf{F}=1$ has the explicit form

$$
F_{11} F_{22}-F_{12} F_{21}=1 \text {. }
$$

We consider a homogeneous anisotropic finite elastic material model based on an isotropic neo-Hookean matrix in which are embedded aligned reinforcing fibres that endow the material with a transversely isotropic character. The model involves the invariants $I_{1}=\operatorname{tr}(\mathbf{C})$ for the neo-Hookean matrix and $I_{5}=\mathbf{A} \cdot\left(\mathbf{C}^{2} \mathbf{A}\right)=\mathbf{a} \cdot(\mathbf{B a})$ related to the reinforcement, where the unit vector $\mathbf{A}$ represents the fibre direction in the undeformed configuration, while its image in the deformed configuration is given by $\mathbf{a}=\mathbf{F A}$. The strain-energy function $W$ of the material is given by

$$
W=\frac{1}{2} \mu\left[I_{1}-3+\rho\left(I_{5}-1\right)^{2}\right]
$$

where $\mu>0$ is the shear modulus of the neo-Hookean matrix material, and $\rho>0$ is the reinforcing strength parameter that regulates the degree of anisotropy.

The fibre direction is now specialized to $\mathbf{A}=\mathbf{E}_{1}$, the square of the stretch in this direction being $I_{4}=\mathbf{A} \cdot(\mathbf{C A})=C_{11}$. Note that the limit $\rho=0$ retrieves the neo-Hookean isotropic base response and $\rho \rightarrow \infty$ corresponds to an inextensible material.

For the considered plane strain deformation $I_{4}$ and $I_{5}$ are related through

$$
I_{5}=C_{11}^{2}+C_{12}^{2}=I_{4}^{2}+C_{12}^{2}
$$

where $C_{12}$ is a measure of shear deformation (fibre shearing).

We denote by $\mathbf{S}$ the first Piola-Kirchhoff stress tensor, which, for a general strain-energy function $W(\mathbf{F})$, is given by

$$
\mathbf{S}=\frac{\partial W(\mathbf{F})}{\partial \mathbf{F}}-p \mathbf{F}^{-\mathrm{T}}
$$

where $p$ is a Lagrange multiplier arising from the incompressibility constraint. Using (5) and (6) the first Piola-Kirchhoff can be expressed as

$$
\mathbf{S}=2 W_{1} \mathbf{F}+2 W_{5}(\mathbf{F A} \otimes \mathbf{C A}+\mathbf{F C A} \otimes \mathbf{A})-p \mathbf{F}^{-\mathrm{T}},
$$

restricted to plane strain as appropriate, where the subscripts 1 and 5 on $W$ refer to the differentiation with respect to $I_{1}$ and $I_{5}$, respectively. The undeformed configuration is 
considered to be stress free so that it follows from (6) that the strain-energy function (3) must satisfy

$$
W=0, \quad 2 W_{1}-p_{0}=0, \quad W_{5}=0
$$

in the reference configuration, where $p_{0}$ is the value of $p$ therein.

From the standard connection the corresponding Cauchy stress $\boldsymbol{\sigma}=\mathbf{S F}^{\mathrm{T}}$ is given by

$$
\boldsymbol{\sigma}=\frac{\partial W(\mathbf{F})}{\partial \mathbf{F}} \mathbf{F}^{\mathrm{T}}-p \mathbf{I}=2 W_{1} \mathbf{B}+2 W_{5}(\mathbf{a} \otimes \mathbf{B a}+\mathbf{B a} \otimes \mathbf{a})-p \mathbf{I},
$$

where $\mathbf{I}$ is the identity tensor.

In the absence of body forces, the equilibrium equations are given in terms of the first Piola-Kirchhoff stress tensor as

$$
\operatorname{Div} \mathbf{S}=\mathbf{0}
$$

\subsection{Piecewise homogeneous deformations: elastostatic shocks}

The solutions of the equilibrium equation for an incompressible nonlinearly elastic fibrereinforced material are assumed to satisfy certain smoothness requirements. If these smoothness requirements are violated then new solutions with different smoothness properties may emerge, as we now discuss. We assume that the system of equations (2) and (9) admits a solution such that $\mathbf{F}, \mathbf{S}$ and $p$ are continuous in $\mathcal{D}$ except on some smooth surface $\mathcal{S}$ across which the deformation and traction are assumed to be continuous but the deformation gradient $\mathbf{F}, \mathbf{S}$ and $p$ are discontinuous. We denote by $\mathcal{S}^{*}$ the image of $\mathcal{S}$ in $\mathcal{D}^{*}$, by $\Pi^{+}$and $\Pi^{-}$the two joined half-spaces in $\mathcal{D}$ and by $\Pi_{*}^{+}$and $\Pi_{*}^{-}$their corresponding images in $\mathcal{D}^{*}$. Let $\left(\mathbf{F}^{+}, \mathbf{S}^{+}, p^{+}\right)$denote the field values in $\Pi^{+}$and $\left(\mathbf{F}^{-}, \mathbf{S}^{-}, p^{-}\right)$those in $\Pi^{-}$. Then, equilibrium in each half-space and traction continuity require

$$
\begin{cases}\operatorname{Div} \mathbf{S}^{ \pm}=\mathbf{0} & \text { in } \mathcal{D}^{ \pm} \backslash \mathcal{S} \\ \llbracket \mathbf{S} \rrbracket_{-}^{+} \mathbf{N}=\mathbf{0} & \text { on } \mathcal{S},\end{cases}
$$

respectively, where $\llbracket \bullet \rrbracket_{-}^{+}=\bullet^{+}-\bullet^{-}$. From $(5)$ the first Piola-Kirchhoff stress tensors in the two half-spaces are given by

$$
\begin{cases}\mathbf{S}^{+}=W_{\mathbf{F}}^{+}-p^{+} \mathbf{F}^{+-\mathrm{T}} & \text { in } \Pi^{+} \\ \mathbf{S}^{-}=W_{\mathbf{F}}^{-}-p^{-} \mathbf{F}^{--\mathrm{T}} & \text { in } \Pi^{-},\end{cases}
$$

where the subscript $\mathbf{F}$ on $W$ signifies differentiation of $W$ with respect to $\mathbf{F}$, with the superscripts \pm indicating evaluation in $\Pi^{ \pm}$.

For the local analysis it is sufficient to consider piecewise homogeneous deformations. Continuity of the deformation then requires

$$
\mathbf{F}^{+} \mathbf{X}=\mathbf{F}^{-} \mathbf{X} \text { on } \mathcal{S},
$$

where $\mathbf{X}=\mathbf{F}^{ \pm} \mathbf{X}$ in $\Pi^{ \pm}$.

More specifically, we consider that the (planar) deformation gradient in $\Pi^{+}$is given by

$$
\mathbf{F}^{+}=\lambda \mathbf{e}_{1} \otimes \mathbf{E}_{1}+\lambda^{-1} \mathbf{e}_{2} \otimes \mathbf{E}_{2},
$$

where the incompressibility constraint (2) has been used. The associated right and left Cauchy-Green deformation tensors, $\mathbf{C}^{+}$and $\mathbf{B}^{+}$, respectively, have diagonal elements

$\left(\lambda^{2}, \lambda^{-2}\right)$. Displacement continuity holds if and only if (see Merodio and Pence, 2001a)

$$
\mathbf{F}^{-}=(\mathbf{I}+k \mathbf{l} \otimes \mathbf{n}) \mathbf{F}^{+},
$$




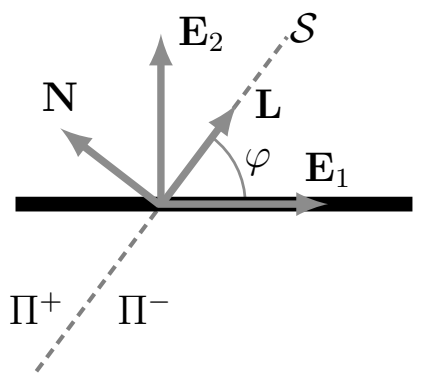

(a) Undeformed configuration $\mathcal{D}$

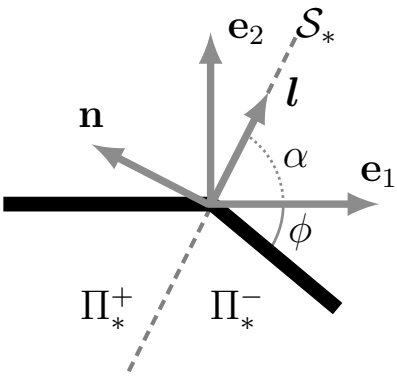

(b) Deformed configuration $\mathcal{D}^{*}$

Figure 1: Kinematics of a plane elastostatic shock: (a) undeformed configuration with the fibre reinforcement direction $\mathbf{E}_{1}$, which is also an in-plane (Lagrangian) principal direction of the deformation, and shock angle $\varphi$; (b) deformed configuration showing the fibre kinking angle $\phi$ and shock angle $\alpha$.

where the parameter $k$ measures the difference between the two deformation gradients, and is known as the shock strength. The main features associated with a shock are shown in Fig. 1. The shock plane is orthogonal to the plane in which the deformation takes place. The fibre reinforcement maintains its initial direction in $\Pi^{+}$, while in $\Pi^{-}$it suffers a change from its initial direction. The angle between the fibre direction in the deformed and undeformed configurations, denoted by $\phi$, is called the kinking angle (see Fig. 1). The dashed line in the undeformed configuration represents the intersection of the elastostatic shock plane $\mathcal{S}$ with the $\left(\mathbf{E}_{1}, \mathbf{E}_{2}\right)$ plane. Let $\mathbf{L}$ and $\mathbf{N}$, respectively, be tangent and the normal unit vectors to the shock line defined by

$$
\mathbf{L}=L_{1} \mathbf{E}_{1}+L_{2} \mathbf{E}_{2}=\cos \varphi \mathbf{E}_{1}+\sin \varphi \mathbf{E}_{2}, \quad \mathbf{N}=N_{1} \mathbf{E}_{1}+N_{2} \mathbf{E}_{2}=-\sin \varphi \mathbf{E}_{1}+\cos \varphi \mathbf{E}_{2} .
$$

The dashed line in the deformed configuration represents the intersection of the elastostatic shock plane $\mathcal{S}^{*}$ with the $\left(\mathbf{e}_{1}, \mathbf{e}_{2}\right)$ plane, and the unit vectors $\mathbf{l}$ and $\mathbf{n}$ are tangential and normal to the shock line, respectively, and given by

$$
\mathbf{l}=l_{1} \mathbf{e}_{1}+l_{2} \mathbf{e}_{2}=\cos \alpha \mathbf{e}_{1}+\sin \alpha \mathbf{e}_{2}, \quad \mathbf{n}=n_{1} \mathbf{e}_{1}+n_{2} \mathbf{e}_{2}=-\sin \alpha \mathbf{e}_{1}+\cos \alpha \mathbf{e}_{2} .
$$

The angles $\varphi$ and $\alpha$ are identified in Fig. 1.

The kinematics of an elastostatic shock for an incompressible material provides a relation between the normal unit vector $\mathbf{n}$ to the shock line in the deformed configuration and its pre-image $\mathbf{N}$ in the undeformed configuration as

$$
\mathbf{N}=|\mathbf{F L}| \mathbf{F}^{\mathrm{T}} \mathbf{n},
$$

which yields a relation between the shock angles $\alpha$ and $\varphi$ via

$$
\tan \alpha=\frac{F_{21}+F_{22} \tan \varphi}{F_{11}+F_{12} \tan \varphi} .
$$

To evaluate $(18)$ one can use either $\mathbf{F}^{+}$or $\mathbf{F}^{-}$to give

$$
\tan \alpha=\lambda^{-2} \tan \varphi \text {. }
$$

In terms of the Cauchy stress tensor, the equilibrium equation $101_{1}$ can be written as $\operatorname{div} \boldsymbol{\sigma}^{ \pm}=\mathbf{0}$ in $\mathcal{D}^{*} \backslash \mathcal{S}^{*}$, and, on use of (17), the traction continuity equation $(10)_{2}$ becomes

$$
\boldsymbol{\sigma}^{+} \mathbf{n}=\boldsymbol{\sigma}^{-} \mathbf{n} \text { on } \mathcal{S}^{*} .
$$


From (13), (14) and (16) the components of $\mathbf{F}^{-}$are given by

$$
F_{11}^{-}=\lambda\left(1+k n_{1} n_{2}\right), \quad F_{22}^{-}=\lambda^{-1}\left(1-k n_{1} n_{2}\right), \quad F_{12}^{-}=\lambda^{-1} k n_{2}^{2}, \quad F_{21}^{-}=-\lambda k n_{1}^{2} .
$$

The associated left Cauchy-Green deformation tensor $\mathbf{B}^{-}$has components

$$
\begin{aligned}
& B_{11}^{-}=\left(1+k n_{1} n_{2}\right)^{2} \lambda^{2}+k^{2} n_{2}^{4} \lambda^{-2}, \quad B_{12}^{-}=-k n_{1}^{2}\left(1+k n_{1} n_{2}\right) \lambda^{2}+k n_{2}^{2}\left(1-k n_{1} n_{2}\right) \lambda^{-2}, \\
& B_{22}^{-}=k^{2} n_{1}^{4} \lambda^{2}+\left(1-k n_{1} n_{2}\right)^{2} \lambda^{-2},
\end{aligned}
$$

and the corresponding components of $\mathbf{C}^{-}$are

$$
\begin{aligned}
& C_{11}^{-}=\left(1+2 k n_{1} n_{2}+k^{2} n_{1}^{2}\right) \lambda^{2}, \quad C_{12}^{-}=k\left(n_{2}^{2}-n_{1}^{2}\right)+k^{2} n_{1} n_{2}, \\
& C_{22}^{-}=\left(1-2 k n_{1} n_{2}+k^{2} n_{2}^{2}\right) \lambda^{-2} .
\end{aligned}
$$

The kinking angle $\phi$ is given by

$$
\tan \phi=\frac{F_{21}^{-}}{F_{11}^{-}}=-\frac{k n_{1}^{2}}{\left(1+k n_{1} n_{2}\right)} .
$$

This framework was considered in Merodio and Pence $(2001 \mathrm{a} b)$ in order to analyze fibre kinking. Fig. 1 gives a local illustration of just one elastostatic shock. The lower part of Fig. 2 is a local illustration (at a point) of the combined kinking and incipient splitting mechanisms with three elastostatic shocks. The upper right part of Fig. 2 shows a kink band given by a three-zone state, denoted $\mathbf{F}^{+} / \mathbf{F}_{\mathrm{K}}^{-} / \mathbf{F}^{+}$, where the index $\mathrm{K}$ refers to the kinking zone. The fibre splitting mechanism also has a three-zone state, denoted $\mathbf{F}^{+} / \mathbf{F}_{\mathrm{S}}^{-} / \mathbf{F}^{+}$, where the index $\mathrm{S}$ refers to the splitting zone.

The angle $\alpha$ gives the direction of the surface of discontinuity (shock). Surfaces normal to the fibre direction are interpreted in terms of fibre kinking. On the other hand, surfaces close to the fibre direction are interpreted in terms of fibre splitting. With this analysis we determine values of $I_{4}$ and $I_{5}$ in both the kinking zone and the splitting zone, which provides information about the mechanisms (all the quantities are evaluated in both the $\Pi^{+}$and $\Pi^{-}$half-spaces). The goal of this work is to characterize both $\mathbf{F}_{\mathrm{K}}^{-}$and $\mathbf{F}_{\mathrm{S}}^{-}$with respect to the considered strain-energy function involving the invariant $I_{5}$.

Continuity of the deformation gives $\mathbf{F}^{-}$in terms of $\mathbf{F}^{+}$and $(k, \alpha)$. Then, for a given deformation gradient $\mathbf{F}^{+}$and a scalar $p^{+}$, one seeks $\left(k, \alpha, p^{-}\right)$satisfying the traction continuity condition (20). Using (3), (13) and (21) in (20), then taking the scalar product of (20) with $\mathbf{n}$ and separately with $\mathbf{l}$, and after some simple manipulations, the traction continuity requirement leads to two equations that can be written as

$$
p^{+}-p^{-}=\mu \rho \sum_{n=1}^{6} a_{n} k^{n}, \quad H=n_{1}^{2} \lambda^{2}+n_{2}^{2} \lambda^{-2}+\rho \sum_{n=0}^{6} b_{n} k^{n}=0,
$$

where, in the latter, the trivial solution $k=0$ has been eliminated. The coefficients $a_{n}$ and $b_{n}$ are given by

$$
\begin{aligned}
& a_{1}=-4 n_{1} n_{2}\left[n_{1}^{2}-n_{2}^{2}-\lambda^{4}\left(3 n_{1}^{2}-n_{2}^{2}\right)+6 \lambda^{8} n_{1}^{2}\right], \\
& a_{2}=4 n_{1}^{2}\left[n_{2}^{2}+6 \lambda^{4} n_{2}^{2}\left(n_{1}^{2}-n_{2}^{2}\right)-3 \lambda^{8} n_{1}^{2}\left(n_{1}^{2}+5 n_{2}^{2}\right)\right], \\
& a_{3}=4 n_{1} n_{2}\left[\left(n_{1}^{2}-n_{2}^{2}\right)^{3}+2 \lambda^{4} n_{1}^{2}\left(n_{1}^{4}+2 n_{1}^{2} n_{2}^{2}-7 n_{2}^{4}\right)-4 \lambda^{8} n_{1}^{4}\left(3 n_{1}^{2}+5 n_{2}^{2}\right)\right], \\
& a_{4}=-4 n_{1}^{2}\left[3 n_{2}^{2}\left(n_{1}^{2}-n_{2}^{2}\right)^{2}+\lambda^{4} n_{1}^{2}\left(n_{1}^{4}-7 n_{1}^{2} n_{2}^{2}+16 n_{2}^{4}\right)+3 \lambda^{8} n_{1}^{4}\left(n_{1}^{2}+5 n_{2}^{2}\right)\right], \\
& a_{5}=12 n_{1}^{3} n_{2}\left[n_{2}^{2}\left(n_{1}^{2}-n_{2}^{2}\right)+\lambda^{4} n_{1}^{2}\left(n_{1}^{2}-3 n_{2}^{2}\right)-2 \lambda^{8} n_{1}^{4}\right], \\
& a_{6}=-4 n_{1}^{4}\left(n_{2}^{2}+\lambda^{4} n_{1}^{2}\right)^{2},
\end{aligned}
$$




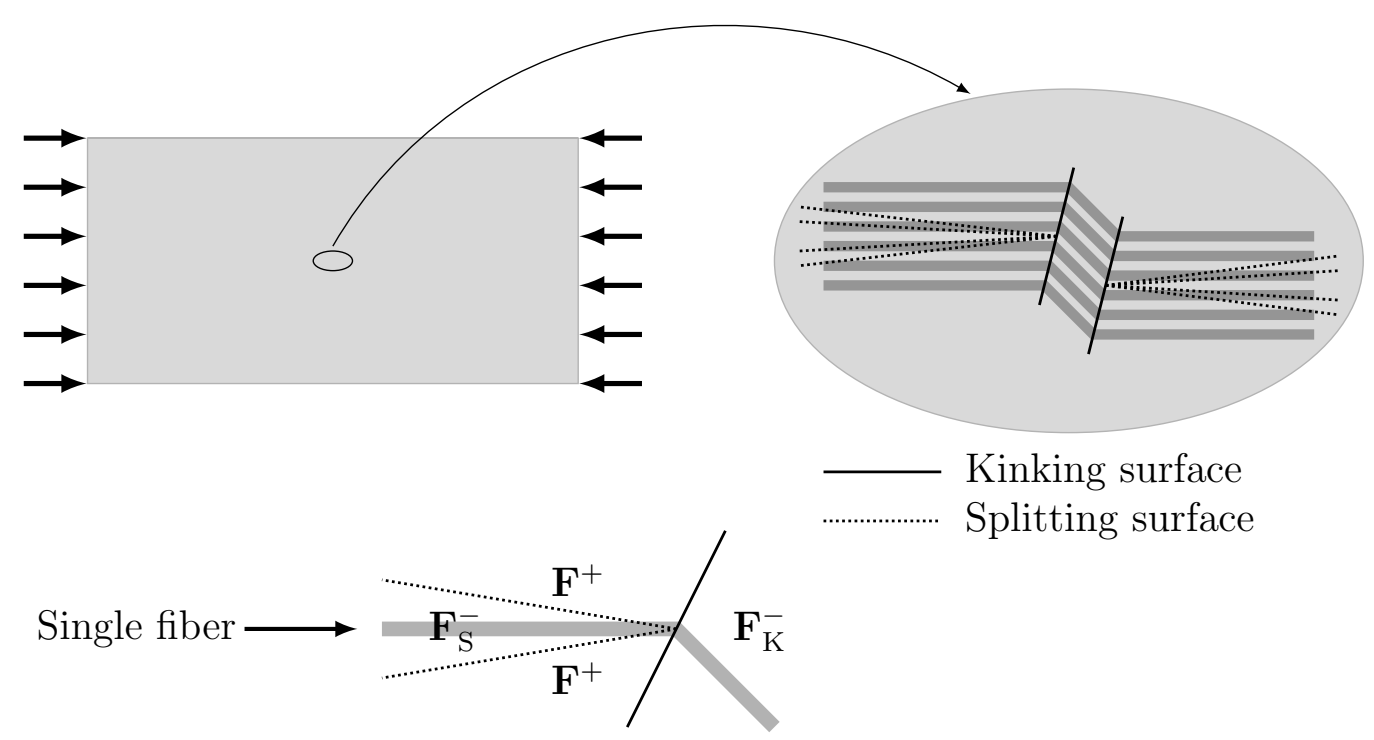

Figure 2: Illustration of fibre kinking and splitting in a unidirectional fibre-reinforced material subject to compressive loading aligned with the initial fibre direction. Fibre kinking is associated with the existence of shocks perpendicular to the fibre reinforcement while fibre splitting is related to the existence of shocks close to the direction of the fibre reinforcement and outside the kink band. More particularly, the kink band is identified by a three-zone state $\mathbf{F}^{+} / \mathbf{F}_{\mathrm{K}}^{-} / \mathbf{F}^{+}$with parallel separating shocks across the first of which the fibres abruptly change direction and then return to the original direction across the second shock. Fibre splitting, which is known to be catastrophic, is illustrated by two symmetrically disposed shocks with respect to the initial fibre direction with a three-zone state $\mathbf{F}^{+} / \mathbf{F}_{\mathrm{S}}^{-} / \mathbf{F}^{+}$.

and

$$
\begin{aligned}
b_{0} & =-2\left[\left(n_{1}^{2}-n_{2}^{2}\right)^{2}+\left(n_{1}^{2}-n_{2}^{2}+8 n_{1}^{2} n_{2}^{2}\right) \lambda^{4}-2 n_{1}^{2}\left(n_{1}^{2}+7 n_{2}^{2}\right) \lambda^{8}\right], \\
b_{1} & =6 n_{1} n_{2}\left[n_{1}^{2}-n_{2}^{2}-\left(n_{1}^{4}+6 n_{1}^{2} n_{2}^{2}-3 n_{2}^{4}\right) \lambda^{4}+2 n_{1}^{2}\left(3 n_{1}^{2}+7 n_{2}^{2}\right) \lambda^{8}\right], \\
b_{2} & =2\left[\left(1-2 n_{1}^{2} n_{2}^{2}\right)\left(1-8 n_{1}^{2} n_{2}^{2}\right)+2 n_{1}^{2}\left(n_{1}^{2}-n_{2}^{2}\right)\left(n_{1}^{4}-6 n_{1}^{2} n_{2}^{2}-15 n_{2}^{4}\right) \lambda^{4}\right. \\
& \left.+2 n_{1}^{4}\left(3 n_{1}^{4}+30 n_{1}^{2} n_{2}^{2}+35 n_{2}^{4}\right) \lambda^{8}\right] \\
b_{3} & =-10 n_{1} n_{2}\left[\left(n_{1}^{2}-n_{2}^{2}\right)^{3}+2 n_{1}^{2} n_{2}^{2}\left(3 n_{1}^{2}-5 n_{2}^{2}\right) \lambda^{4}-2 n_{1}^{4}\left(3 n_{1}^{2}+7 n_{2}^{2}\right) \lambda^{8}\right], \\
b_{4} & =6 n_{1}^{2}\left[3 n_{2}^{2}\left(n_{1}^{2}-n_{2}^{2}\right)^{2}+n_{1}^{2}\left(n_{1}^{2}-3 n_{2}^{2}\right)\left(n_{1}^{2}-5 n_{2}^{2}\right) \lambda^{4}+2 n_{1}^{4}\left(n_{1}^{2}+7 n_{2}^{2}\right) \lambda^{8}\right], \\
b_{5} & =-14 n_{1}^{3} n_{2}\left[n_{2}^{2}\left(n_{1}^{2}-n_{2}^{2}\right)+n_{1}^{2}\left(n_{1}^{2}-3 n_{2}^{2}\right) \lambda^{4}-2 n_{1}^{4} \lambda^{8}\right], \\
b_{6} & =4 n_{1}^{4}\left(n_{2}^{2}+n_{1}^{2} \lambda^{4}\right)^{2} .
\end{aligned}
$$

For a given deformation gradient $\mathbf{F}^{+}$and material parameter $\rho$, it follows that if $\left(\lambda, n_{1}, n_{2}, k\right)$ is a solution of (25) then $\left(\lambda,-n_{1}, n_{2},-k\right)$ and $\left(\lambda, n_{1},-n_{2},-k\right)$ are also solutions since $H\left(\lambda,-n_{1}, n_{2},-k\right)=H\left(\lambda, n_{1}, n_{2}, k\right)$. Hence, noting that in this paper $\alpha$ is measured in degrees, one may restrict attention to $\alpha \in[0,90]$. This is in agreement with the fibre kinking and splitting mechanism illustrated in the lower part of Fig. 2. For $k$ associated with the solution $\left(\lambda, n_{1}, n_{2}, k\right)$ there also exists a solution $\left(\lambda, n_{1},-n_{2},-k\right)$. Before a more general characterization of equilibrium shocks, we consider several special shocks that will inform the subsequent analysis. In particular, in the next section we consider weak shocks for which $(k \rightarrow 0)$, orthogonal shocks $(\alpha=90)$ and parallel shocks $(\alpha=0)$. Stability is not an immediate matter of concern, but will be treated later on in Section 5 . 


\section{Some special elastostatic shocks}

\subsection{Weak elastostatic shocks}

The governing differential equation changes locally in type from elliptic to hyperbolic as the deformation of the material proceeds. This change is referred to as loss of ordinary ellipticity and it leads to the emergence of weak surfaces of discontinuity (otherwise referred to as weak shocks or characteristic surfaces). Let $\xi$ be a coordinate in the direction normal to the shock. The surfaces carrying the discontinuities of the deformation gradient $\mathbf{F}$, the stress tensor $\mathbf{S}$ and the pressure $p$ are often referred to as strong elastostatic shocks. On the other hand, weak elastostatic shocks involve a continuous derivative $\partial \mathbf{u} / \partial \xi$ of the displacement field as well as a continuous pressure $p$, but a discontinuous second derivative $\partial^{2} \mathbf{u} / \partial \xi^{2}$ of the displacement field $\mathbf{u}$ and a discontinuous first derivative $\partial p / \partial \xi$ of $p$. We now show that a strong elastostatic shock turns into a weak elastostatic shock as the shock strength tends to zero. The loss of ellipticity is given by (see, for example, Merodio and Ogden, 2002)

$$
\mathrm{Q}(\mathbf{n}): \mathbf{l} \otimes \mathbf{l}=\mathbf{0}
$$

where

$$
Q_{i j}=\mathrm{F}_{p \alpha} \mathrm{F}_{q \beta} \frac{\partial^{2} W}{\partial \mathrm{F}_{j \beta} \mathrm{F}_{i \alpha}} n_{p} n_{q}
$$

are the components of the acoustic tensor $\mathbf{Q}$ and, subject to $\mathbf{l} \cdot \mathbf{n}=0, \mathbf{l}$ and $\mathbf{n}$ are arbitrary unit vectors as distinct from the specific $\mathbf{l}$ and $\mathbf{n}$ introduced in Section 2.2, and : signifies double contraction.

The analysis of $(28)$ for a given $W$ furnishes the ellipticity status of that particular strain energy. If, for some pair of orthogonal unit vectors $\mathbf{l}$ and $\mathbf{n}$ such that $\mathbf{l} \cdot \mathbf{n}=0$, a given deformation gradient $\mathbf{F}$ satisfies equation (28), then the deformation is said to be non-elliptic for that material model. Furthermore, the unit vector $\mathbf{n}$ is then identified as the normal vector to a surface (in the deformed configuration), referred to as a weak surface of discontinuity while $\mathbf{l}$ is in the direction of the tangent to the surface, following the terminology introduced in the previous section. Once $\mathbf{F}$ is specified, it is possible to check the ellipticity status of the deformation (see Merodio and Neff, 2006).

In respect of (3) the ellipticity condition (28) specializes to

$$
\begin{aligned}
& \left\{(\mathbf{B}: \mathbf{n} \otimes \mathbf{n}) \mathbf{I}+2 \rho\left(I_{5}-1\right)[2(\mathbf{B}: \mathbf{a} \otimes \mathbf{n})(\mathbf{a} \cdot \mathbf{n}) \mathbf{I}+(\mathbf{B}: \mathbf{n} \otimes \mathbf{n})(\mathbf{a} \otimes \mathbf{a})\right. \\
& \left.+(\mathbf{a} \cdot \mathbf{n})(\mathbf{a} \otimes \mathbf{B n})+(\mathbf{a} \cdot \mathbf{n})(\mathbf{B n} \otimes \mathbf{a})+(\mathbf{a} \cdot \mathbf{n})^{2} \mathbf{B}\right] \\
& +4 \rho[(\mathbf{B}: \mathbf{a} \otimes \mathbf{n}) \mathbf{a}+(\mathbf{a} \cdot \mathbf{n}) \mathbf{B a}] \otimes[(\mathbf{B}: \mathbf{a} \otimes \mathbf{n}) \mathbf{a}+(\mathbf{a} \cdot \mathbf{n}) \mathbf{B a}]\}: \mathbf{l} \otimes \mathbf{l}=\mathbf{0},
\end{aligned}
$$

which, on use of (13) and (16), becomes

$$
4 \rho\left(7 n_{2}^{2}+n_{1}^{2}\right) n_{1}^{2} \lambda^{8}-2 \rho\left(n_{1}^{2}-n_{2}^{2}+8 n_{1}^{2} n_{2}^{2}\right) \lambda^{4}+n_{1}^{2} \lambda^{2}+2 \rho\left(4 n_{1}^{2} n_{2}^{2}-1\right)+n_{2}^{2} \lambda^{-2}=0 .
$$

This identifies weak surfaces of discontinuity characterised by $(\lambda, \alpha)$ for a fixed material parameter $\rho$. It is easy to show that $(30)$ is equivalent to the general equation $(25)_{2}$ that gives elastostatic shocks in the limit $k \rightarrow 0$ for which $n_{1}^{2} \lambda^{2}+n_{2}^{2} \lambda^{-2}+\rho b_{0}=0$. Our purpose now is to determine the emergence of these weak surfaces of discontinuity. Under compressive loading, attention is restricted to $\lambda \in(0,1)$ and, by symmetry, to $\alpha \in[0,90]$. When $\rho$ is sufficiently large, ellipticity is lost for $\lambda=\lambda_{o}<1$, but very close to the undeformed configuration and the weak surface is perpendicular to the fibre $(\alpha=90)$, which is interpreted as fibre kinking. Hence, very moderate fibre compression is sufficient for ellipticity loss. As $\lambda$ decreases (inside the non-elliptic region) there could be one weak 
(a)

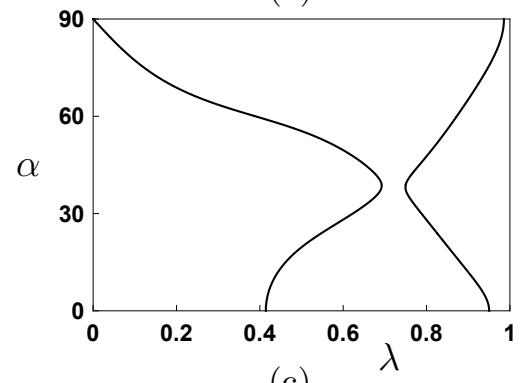

(c)

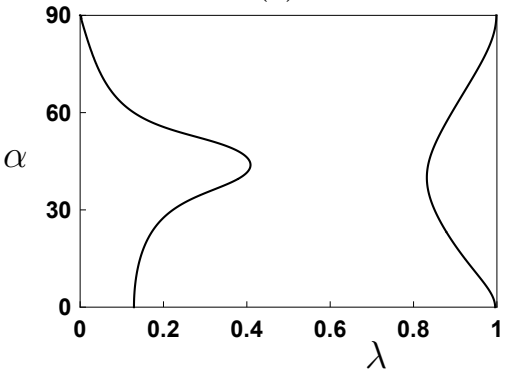

(b)

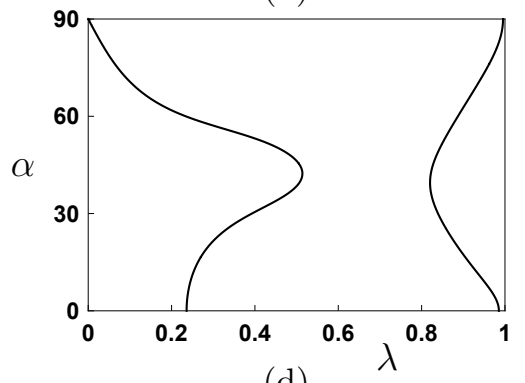

(d)

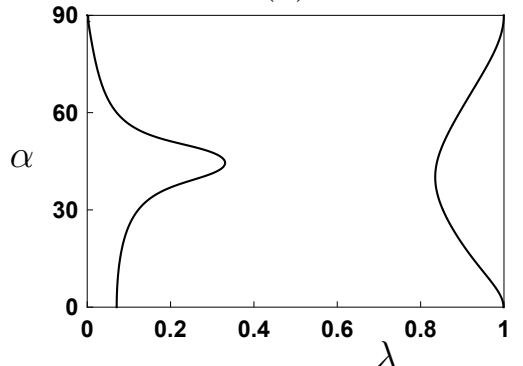

Figure 3: The curves in each panel show plots of $\alpha$ versus $\lambda$ based on $(29)$ for $\rho=3,9,30,60$ in (a)-(d), respectively, thus providing their ellipticity status. In each panel, the right-hand curve is interpreted as follows. The incipient loss of ellipticity occurs for $\lambda=\lambda_{o}<1$ (close to the undeformed configuration $\lambda=1$ ) and the associated weak surface is perpendicular to the fibre, i.e. $\alpha=90$, which is interpreted as fibre kinking. As $\lambda$ decreases (in the non-elliptic domain) the curve gives one weak surface satisfying $\alpha<90$ until $\lambda=\lambda_{p}$ for which it is possible, in addition, to obtain a weak surface parallel to the fibre, i.e. $\alpha=0$, which is interpreted as fibre splitting. The left-hand curve in each plot is not of interest since ellipticity has already been lost.

surface for which $\alpha<90$. Furthermore, it is possible to obtain a second weak surface parallel to the fibre with $\alpha=0$ for $\lambda=\lambda_{p}$, which is interpreted as fibre splitting. The emergence of these two solutions, and the results obtained here, are in agreement with those given by Merodio and Ogden (2002). These results are summarized in Fig. 3. The values $\lambda_{o}$ and $\lambda_{p}$ depend on the material parameter $\rho$. Table 1 shows these values for a selection of specific values of $\rho$.

\begin{tabular}{ccccccc}
\hline \hline$\rho$ & 1 & 1.3 & 3 & 9 & 30 & 60 \\
\hline$\lambda_{o}$ & 0.9541 & 0.9655 & 0.9856 & 0.9953 & 0.9986 & 0.9993 \\
$\lambda_{p}$ & - & 0.7682 & 0.9502 & 0.9853 & 0.9957 & 0.9979 \\
\hline \hline
\end{tabular}

Table 1: Values of $\lambda_{o}$ associated with the incipient shock $\alpha=90$ and $\lambda_{p}$ associated with the incipient shock $\alpha=0$ for $\rho=1,1.3,3,9,30,60$.

If there exists a $(\lambda, \alpha)$ pair satisfying (30) for a fixed material parameter $\rho$, then a weak surface of discontinuity (weak shock) may arise inside the material. Strong surfaces of discontinuity (strong shocks) described by the set of parameters $(\lambda, k, \alpha)$, in which $k$ represents the strength of the shock may also arise inside the material. Based on the weak shocks obtained, we now focus attention on particular strong shocks: those satisfying $\alpha=90$, which are called orthogonal strong surfaces of discontinuity (orthogonal strong shocks), and those satisfying $\alpha=0$, which are referred to as parallel strong surfaces of discontinuity (parallel strong shocks). 


\subsection{Orthogonal strong shocks}

In this section attention is restricted to orthogonal strong shocks. Using $\alpha=90,(25)_{2}$ and (27) one obtains a cubic in $k^{2}$ :

$$
4 \rho \lambda^{8} k^{6}+6 \rho \lambda^{4}\left(2 \lambda^{4}+1\right) k^{4}+2 \rho\left(2 \lambda^{4}+6 \lambda^{8}+1\right) k^{2}-2 \rho\left(1+\lambda^{4}-2 \lambda^{8}\right)+\lambda^{2}=0 .
$$

By applying Descarte's rule of signs, it is easy to check that 31) admits non-zero real solutions for $k$ provided

$$
\rho>\frac{\lambda^{2}}{2\left(1+\lambda^{4}-2 \lambda^{8}\right)}=\bar{\rho}(\lambda),
$$

wherein $\bar{\rho}$ is defined. Fig. 4 shows values of $\bar{\rho}$ versus $\lambda$ from (32), obtained from (31) with $k=0$.

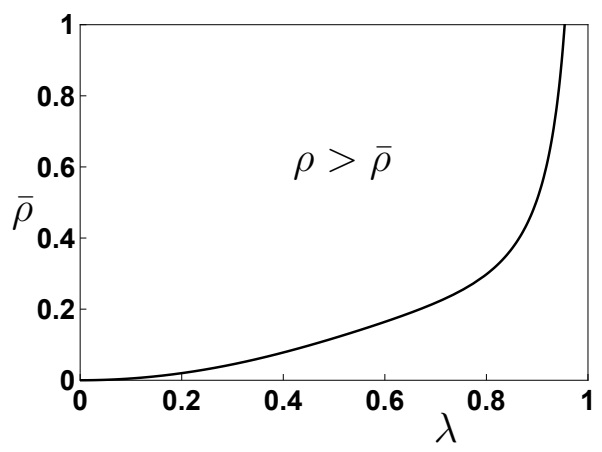

Figure 4: Plot of $\bar{\rho}(\lambda)$ given by (32). For a given value of $\lambda$, orthogonal shocks are only possible in the region above the curve defined by $\rho>\bar{\rho}(\lambda)$.

According to these results, (31) admits real solutions if and only if $\rho>\bar{\rho}(\lambda)$. Note that for $\rho=0$ an orthogonal strong surface is not possible. Fig. 5 shows values of the shock strength $k$ against $\lambda$ satisfying (31) for several values of $\rho$ for which (32) holds. In summary, for a value of the reinforcing parameter $\rho$ satisfying (32), a weak solution emerges when $\lambda=\lambda_{o}$ as $\lambda$ decreases from $\lambda=1$. A further decrease in $\lambda$ develops the weak orthogonal shock into a strong orthogonal shock.

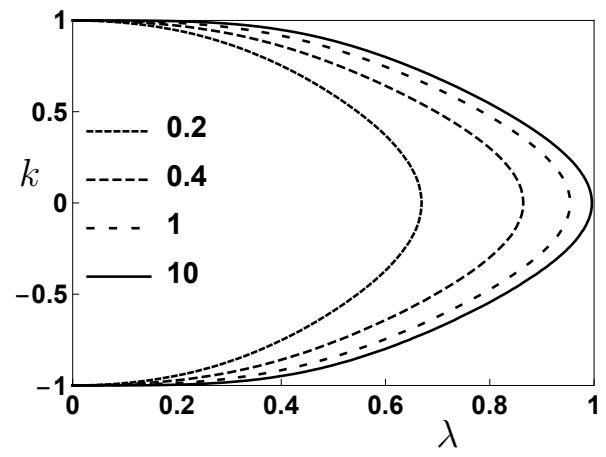

Figure 5: Plots of $k$ versus $\lambda$ from (31), which are associated with strong orthogonal shocks for the indicated values of $\rho=0.2,0.4,1,10$. As $\lambda$ decreases from 1 , the first shock that emerges in each curve is a weak shock, i.e. $k=0$. The weak shock further develops into a strong shock with decreasing values of $\lambda$. The curves are symmetric with respect to the $\lambda$ axis. In addition, using (24), it is easy to show that if $k<0(k>0)$ then $\phi>0(\phi<0)$. 
We now examine different kinematical variables in the two zones of the localized deformation for a given value of $\rho$. In $\Pi^{+}$the deformation is given by $\lambda$. For $\lambda<1$ it follows that $I_{4}^{+}<1$ and $I_{5}^{+}<1$ since $I_{5}^{+}=\left(I_{4}^{+}\right)^{2}=\lambda^{4}$. For such a $\lambda$, the deformation in $\Pi^{-}$is provided by values $(\lambda, k, \alpha=90)$ satisfying (31). Values of $k$ can be taken either positive or negative since this only affects the fibre kinking direction, i.e. the sign of the fibre shearing. We focus on values $k<0$ for which, by $(23)_{2}, C_{12}^{-}>0$. In particular, $I_{4}^{-}=C_{11}^{-}$is obtained using (23) $)_{1}$ and $C_{12}^{-}$is obtained using $(23)_{2}$. These two values, in turn, using (4), give $I_{5}^{-}$. In Fig. 6 the quantities $\left(C_{11}^{-}\right)^{1 / 2}$ (fibre stretch), $C_{12}^{-}$(fibre shearing), $I_{4}^{-}$and $I_{5}^{-}$are plotted against $\lambda$. We plot all the quantities for completeness and clarity since although they are related it is important to single out their values to fully understand their influence on the different fibre instabilities that are exhibited later on during the energetic analysis. At this point we just mention that the fibre in $\Pi^{-}$is subject to both shearing and contraction.
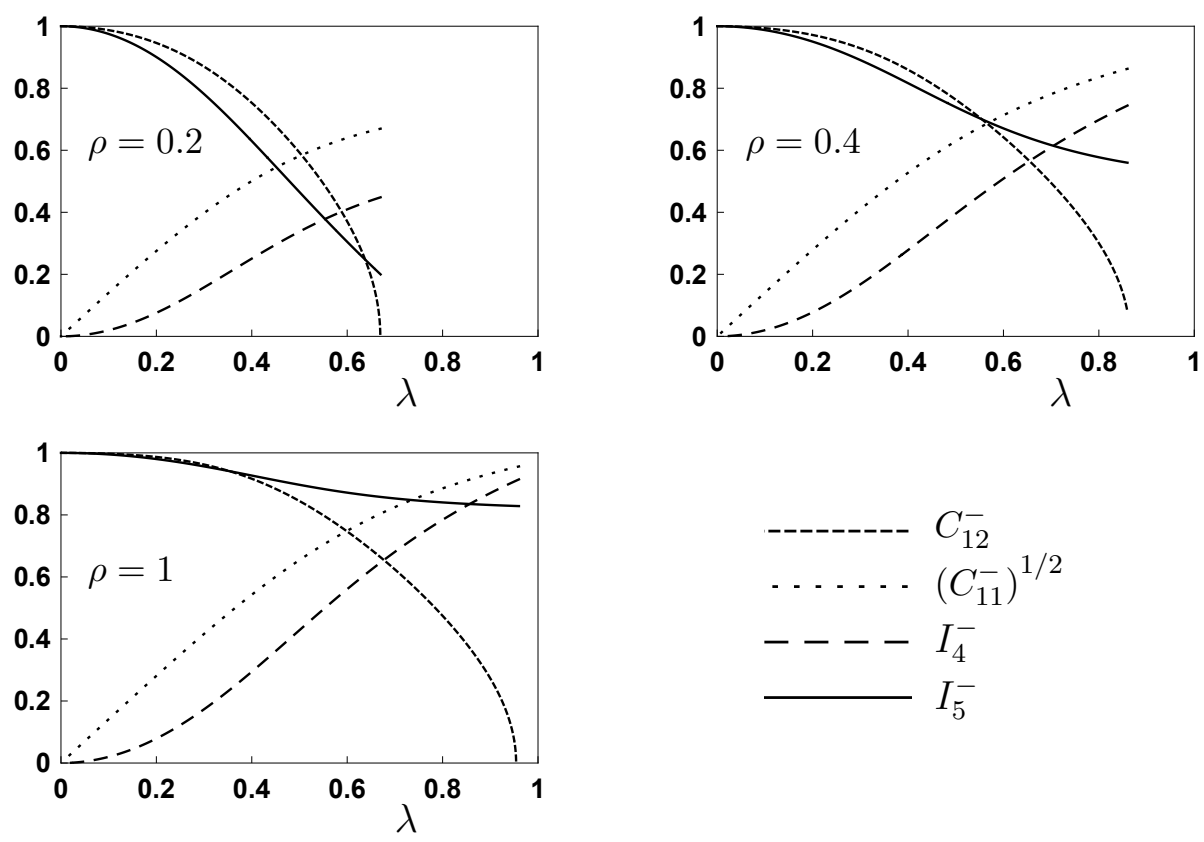

Figure 6: Plots of $\left(C_{11}^{-}\right)^{1 / 2}, C_{12}^{-}, I_{4}^{-}$and $I_{5}^{-}$versus $\lambda$ for $\rho=0.2,0.4,1$. These values provide the deformation in $\Pi^{-}$for orthogonal strong shocks. For any value $\rho$, as $\lambda$ decreases $C_{12}^{-}$increases monotonically up to $C_{12}^{-} \approx 1$ while $C_{11}^{-}$decreases monotonically down to $C_{11}^{-} \approx 0$.

\subsection{Parallel strong shocks}

Parallel strong shocks are given by 25$)_{2}$ with 27$)$ restricted to $\alpha=0$, which yields

$$
k^{2}-\frac{1}{2 \rho \lambda^{2}}\left(-2 \rho \lambda^{6}+2 \rho \lambda^{2}-1\right)=0,
$$

from which it follows that a necessary condition for such a surface to exist is

$$
\rho>\frac{1}{2 \lambda^{2}\left(1-\lambda^{4}\right)}=\hat{\rho}(\lambda)
$$

which defines $\hat{\rho}$.

In Fig. 7 the curve gives the values $\hat{\rho}(\lambda)$ versus $\lambda$ that satisfy (34). A parallel strong shock exists if and only if $\rho>\hat{\rho}^{*}$, where $\hat{\rho}^{*} \approx 1.298$. Alternatively, for such a value of $\rho$, 


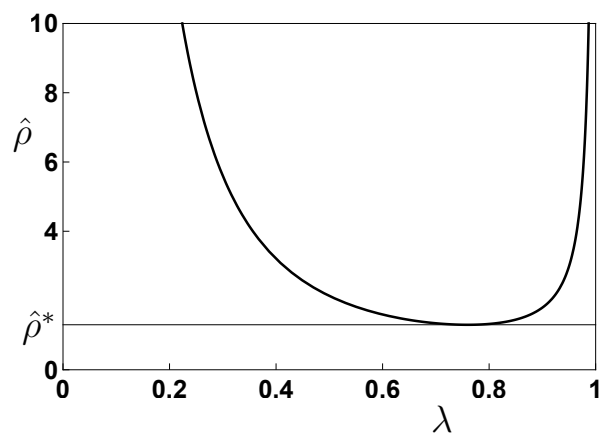

Figure 7: The curve shows values of $\hat{\rho}(\lambda)$ from (34). Its minimum occurs for $\rho=\hat{\rho}^{*} \approx 1.298$. For a given $\rho>\hat{\rho}^{*}$, the curve gives two values of $\lambda$ : the minimum value is referred to as $\lambda_{\text {min }}$ and the maximum is called $\lambda_{\max }$. Parallel strong shocks only exist for values $\lambda \in\left[\lambda_{\min }, \lambda_{\max }\right]$. Furthermore, $\lambda_{\max }=\lambda_{p}$.

parallel strong shocks are possible for values of $\lambda \in\left[\lambda_{\min }, \lambda_{\max }\right]$, where $\lambda_{\min }$ and $\lambda_{\max }$ are the $\rho$-dependent minimum and maximum values of $\lambda$ from $\rho=1 / 2 \lambda^{2}\left(1-\lambda^{4}\right)$. Furthermore, it follows that $\lambda_{\max }=\lambda_{p}$. Hence, for a value of the reinforcing parameter $\rho$ satisfying (34) as $\lambda$ decreases from $\lambda=1$ a parallel weak solution emerges when $\lambda=\lambda_{p}$. A further decrease in $\lambda$ develops the weak parallel shock into a strong one.

In Fig. 8 values of the strength of the shock $k$ given by (33) against $\lambda$ for specific values of the reinforcing parameter $\rho$ are shown. As $\lambda$ decreases from $\lambda=1$, the first solution of (33) is a weak solution, i.e. $k=0$. Solutions with $k \neq 0$ emerge as $\lambda$ decreases further. Values of $k$ can be either positive or negative, which affects only the fibre kinking direction, i.e. the sign of the fibre shearing. From (23), we have $C_{12}^{-}=k$ and $I_{4}^{-}=C_{11}^{-}=\lambda^{2}$ and, on use of the expression (4) and (33), $I_{5}^{-}=1-1 /\left(2 \rho \lambda^{2}\right)$. Clearly, the fibre stretch $\left(C_{11}^{-}\right)^{1 / 2}$ depends linearly on $\lambda$, while the fibre shearing $C_{12}^{-}=k$ is given by (33), all subject to the $\rho$-dependent restriction $\lambda \in\left[\lambda_{\min }, \lambda_{\max }\right]$.

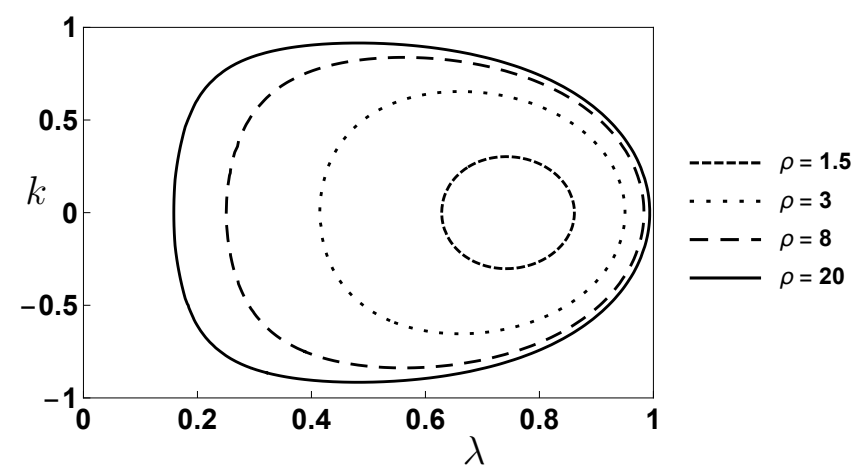

Figure 8: Plots of $k$ versus $\lambda$ from (31) that correspond to strong parallel surfaces for the material parameter $\rho=1.5,3,8,20$.

Clearly, $I_{4}^{-}<1$ for all $\lambda$ in the latter range, and as $\lambda$ decreases from $\lambda=\lambda_{\max }=\lambda_{p}$ the fibre continues to contract while initially shearing takes place with the shear component $k=\mathrm{C}_{12}^{-}$increasing from 0 at $\lambda_{\max }$ until it reaches a maximum, thereafter decreasing down to 0 again at $\lambda=\lambda_{\min }$. This is in contrast to the case of an orthogonal strong surface (see Fig. 6) for which $C_{12}^{-}$increases from zero at $\lambda=\lambda_{o}$. Note that $I_{5}^{-}>0$ requires $2 \rho \lambda^{2}>1$ and hence $I_{5}^{-}<1$ in the considered range. 


\section{The general solution for elastostatic shocks}

In this section, for a fixed reinforcing parameter $\rho$, a general three-dimensional solution in $(\lambda, k, \alpha)$ space of $(25)_{2}$ is determined and examined. It is first illustrated for $\rho=3$ since for larger values of $\rho$, the main features of the solution have similar characteristics. The projection of the solution for $\rho=3$ on to the $(\lambda, k)$ plane is shown in Fig. 9, which reflects the symmetry $\left(\lambda, n_{1}, n_{2}, k\right) \leftrightarrow\left(\lambda,-n_{1}, n_{2},-k\right)$ about the horizontal line at $k=0$.

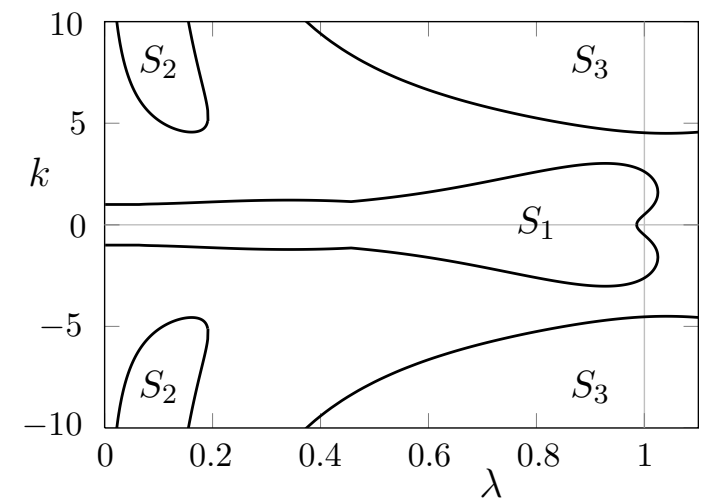

Figure 9: Plot of the projection of the three-dimensional solution $(\lambda, k, \alpha)$ of 25$)_{2}$ on to the $(\lambda, k)$ plane for $\rho=3$. There are no solutions in the region that is not labelled. The two families of solutions, $S_{2}$ and $S_{3}$, are associated with angles $\alpha \approx 90$ and $\alpha \approx 0$, respectively, which can be interpreted in terms of orthogonal and parallel strong surfaces of discontinuity.

The solutions $S_{2}$ and $S_{3}$ are associated with angles $\alpha \approx 90$ and $\alpha \approx 0$, respectively. They can be interpreted in terms of orthogonal and parallel strong shocks. Fig. 9 shows the complete domain of the solutions $(\lambda, k, \alpha)$ associated with mechanically consistent strong shocks as given by $(25)_{2}$ for $\rho=3$. Note that $(25)_{2}$ admits real solutions for which $\lambda>1$, but these will be ruled out later on by energy considerations.

Let us consider, without loss of generality, some of the solutions labelled $S_{1}$, in particular those for which $\alpha \in[0,90]$. Fig. 10 shows the solution of $(25)_{2}$ as a surface in the threedimensional space $(\lambda, k, \alpha)$. The projection of this surface on to the $(\lambda, k)$ plane is not the whole area identified as $S_{1}$ since, by symmetry, the area $S_{1}$ captures all solutions, not just those for $\alpha \in[0,90]$. The solutions in $S_{1}$ indicate that if a solution exists for the pair of values $(\lambda, k)$, then $(\lambda,-k)$ is also a solution. The values of $(\lambda, k, \alpha)$ on the surface in Fig. 10 give rise to shocks. The curve where the surface shown in Fig. 10(a) intersects the plane $\alpha=90$ has the same shape as the curves shown in Fig. 5 for different values of $\rho$, while the curve where the surface shown in Fig. 10(b) intersects the plane $\alpha=0$ is that given in Fig. 8 for $\rho=3$, thus connecting this analysis with the solutions obtained in the previous section.

For larger values of $\rho$ the families of solutions $S_{1}$ and $S_{3}$ intersect at some point in the $(\lambda, k)$ plane as well as do $S_{1}, S_{2}$ and $S_{3}$. This is illustrated in Fig. 11 for $\rho=9,30$. 
(a)

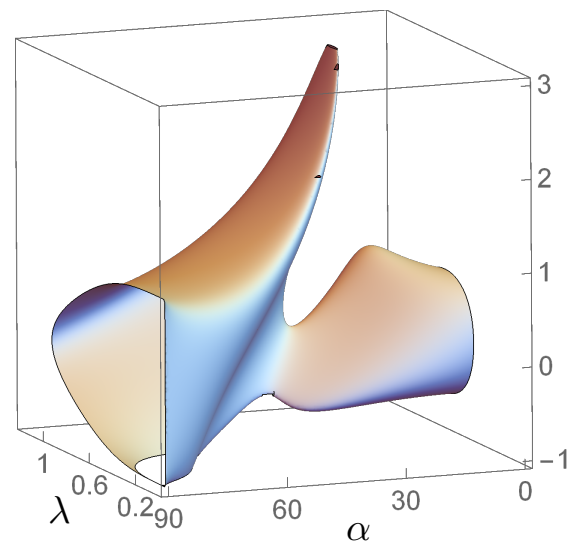

(b)

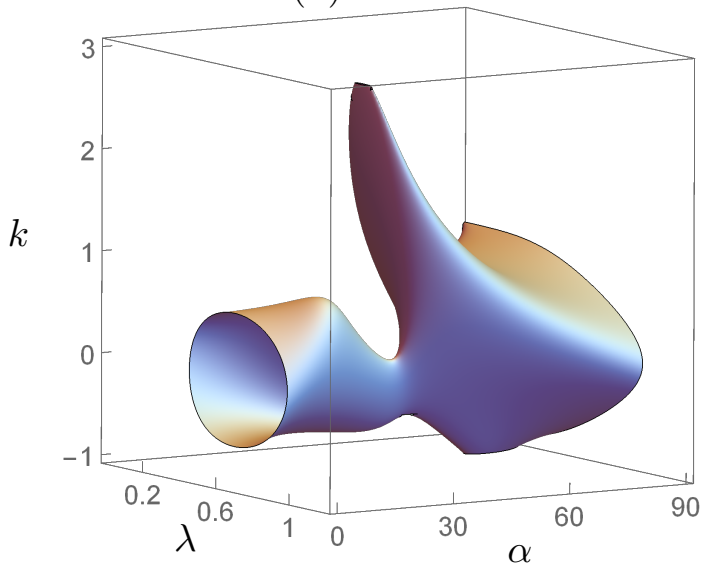

Figure 10: The three-dimensional solution $(\lambda, k, \alpha)$ for $\alpha \in[0,90]$ associated with the solutions shown in $S_{1}$, as given by $25{ }_{2}$ for $\rho=3$ from two different viewing positions.

(a)

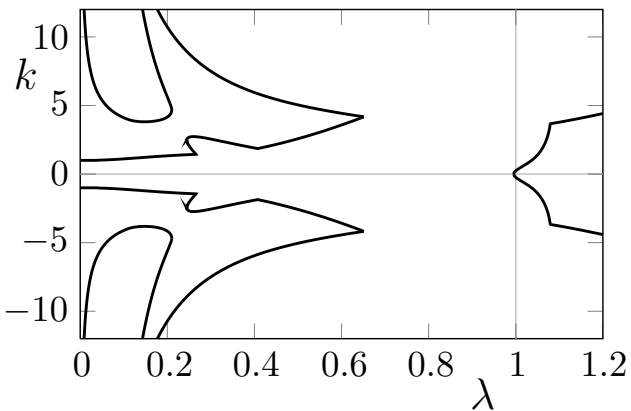

(b)

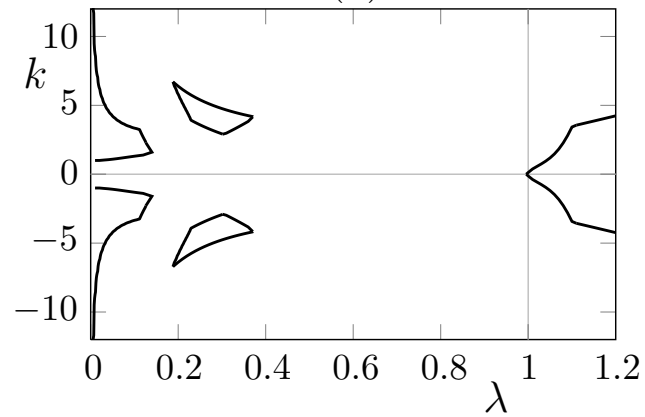

Figure 11: The figures illustrate the projection of the three-dimensional solution $(\lambda, k, \alpha)$ of $(25)_{2}$ on to the $(\lambda, k)$ plane for $\rho=9$ and $\rho=30$ in (a) and (b), respectively. A visual comparison of Fig. 9 and Fig. 11 shows that as $\rho$ increases, the areas $S_{i}, i=1,2,3$, in Fig. 9 intersect. In particular, for $\rho=9$, the areas $S_{1}$ and $S_{3}$ intersect, while for $\rho=30$ all three areas intersect.

For $\rho=3$ several cross sections of the solution of $(25)_{2}$ for different values of $\lambda$, i.e. $\lambda=1,0.9856,0.8$ and 0.4 , are shown in Fig. 12 .

This gives a representation of the mechanically consistent equilibrium kinks associated with a specific value of $\rho$ and $\mathbf{F}^{+}$. Each cross section shows that if $(\lambda, \alpha, k)$ is a solution then $(\lambda, 180-\alpha,-k)$ is also a solution. For $\lambda=1>\lambda_{p}=0.9856$ the cross sections consist of four distinct components. From the symmetry $(\lambda, \alpha, k) \leftrightarrow(\lambda, 180-\alpha,-k)$ we just consider the two components (curves) for which $\alpha \in[0,90]$. The projection of the component (curve) at the top left corner on to the $(\lambda, k)$ plane is related to values in the area denoted $S_{3}$ in Fig. 9. The projection of the other curve onto the $(\lambda, k)$ plane is related to values in the area denoted $S_{1}$. Furthermore, points $(\lambda, k, \alpha)$ of this curve are on the surface of the threedimensional solution shown in Fig. 10. The projections of the corresponding symmetric components in that cross section on to the $(\lambda, k)$ plane follow from the symmetry of the solutions. For $\lambda=\lambda_{o}=0.9856$ the cross sections consist of four distinct components, but two of these components coalesce (at $\alpha=90$ ), and, furthermore, as $\lambda$ decreases these two components merge into a single component. For $\lambda=0.8$ two new (additional) components are introduced. These solutions are related to parallel shocks, which emerge as weak shocks when $\lambda=\lambda_{p}=0.9502$. The projection of these two components on to the $(\lambda, k)$ plane is related to values in the area denoted $S_{1}$ in Fig. 9. For $\lambda=0.4$ the cross section has only 
(a)
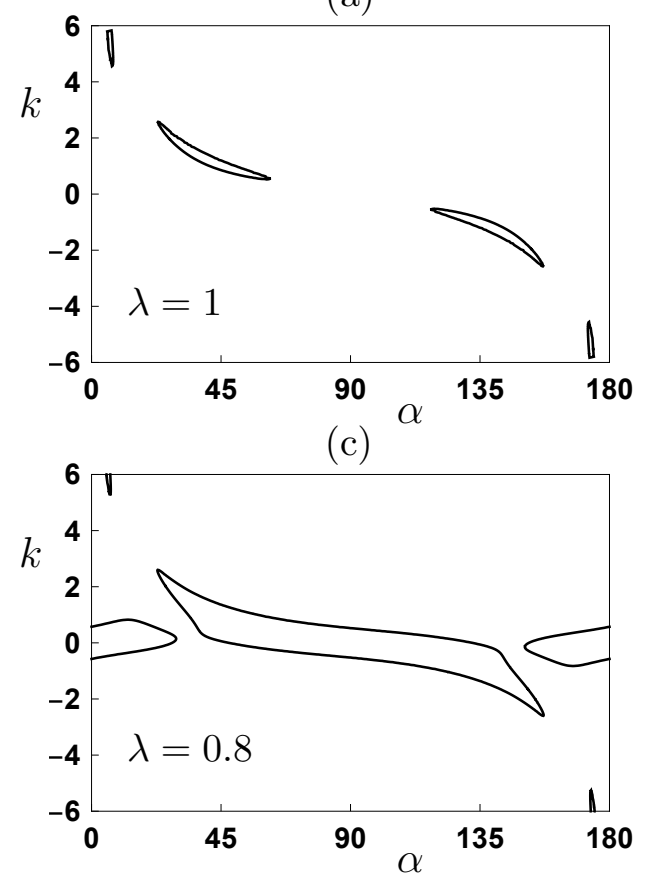

(b)

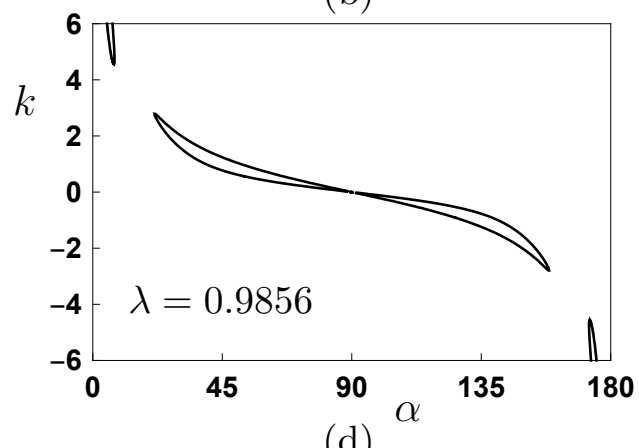

(d)

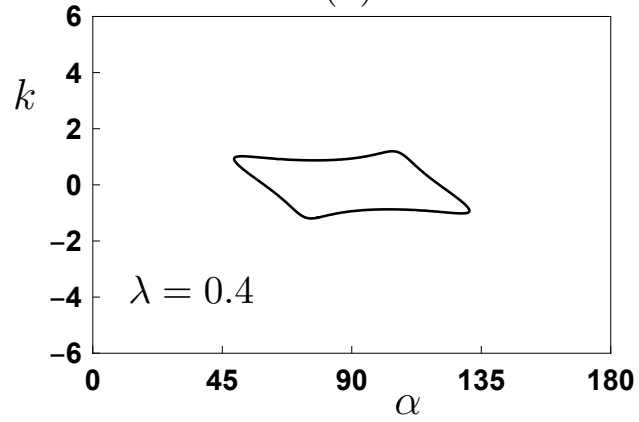

Figure 12: Different $\lambda$ cross sections of the solution of $(25)_{2}$ for $\rho=3$.

one component. For smaller values of $\lambda$, for instance $\lambda=0.1$, the cross sections include values related to the area denoted as $S_{2}$ in Fig. 9. These values do not attract our attention since they are related to excessive (fibre) compression.

For $\rho=30$ some $\lambda$ cross sections of the solution of $(25)_{2}$ are shown in Fig. 13 for completeness. In particular, four specific cross sections corresponding to $\lambda=0.999,0.98$, 0.93 and 0.92 are shown. All cross sections show that if $(\lambda, \alpha, k)$ is a solution then $(\lambda, 180-$ $\alpha,-k)$ is also a solution. For $\lambda=0.999>\lambda_{o}=0.9986$ the cross section consists of the four distinct components described when $\lambda=1$ for $\rho=3$ and additionally there are two (new) components. These two solutions are related to parallel shocks. They will contact the point $(k=0, \alpha=0)$ when $\lambda_{p}=0.9957$ and for smaller values of $\lambda$ these components develop as illustrated for $\lambda=0.98$, which is then described similarly to Fig. 12(c). The other two cross sections show how the different components intersect.

\subsection{Ellipticity status of the shocks and half-spaces}

An equilibrated elastostatic shock is possible inside the material if and only if, for a given $\rho$, there exists a set $(\lambda, k, \alpha)$ that satisfies $(25)_{2}$. We show here that the loss of ordinary ellipticity is a necessary condition for such an elastostatic shock to arise.

In this section the focus is on the type of deformation in the two half-spaces for a mechanically consistent strong shock. The deformations in $\Pi^{+}$and $\Pi^{-}$may be either elliptic or non-elliptic. If that in $\Pi^{+}$is elliptic or non-elliptic, then that in $\Pi^{-}$may be either elliptic or non-elliptic (see Merodio, 1999; Merodio and Pence, 2001a b). Recall that the deformation in $\Pi^{+}$is characterized by $\lambda$ through (13) and that in $\Pi^{-}$is characterized by $\lambda, k$ and $\alpha$ by means of (21). Since weak shocks require loss of ordinary ellipticity in $\Pi^{+}$under the deformation in (13) and involve $\mathbf{F}^{-}=\mathbf{F}^{+}$, then weak shocks involve the loss of ordinary ellipticity in $\Pi^{-}$under the deformation given in (21). The existence of strong shocks $(k \neq 0)$ can be associated with two different elliptic deformation gradients (see 
(a)

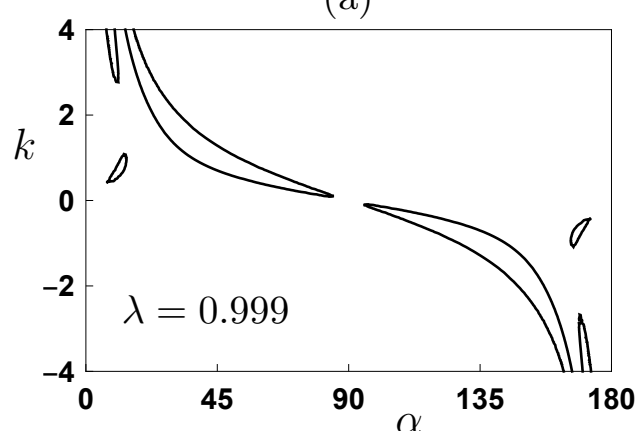

(c)

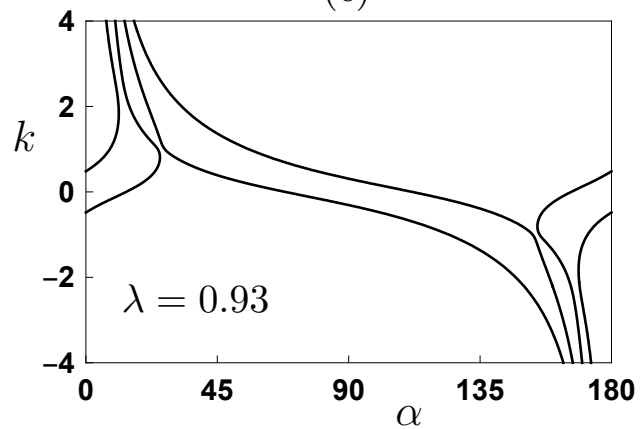

(b)

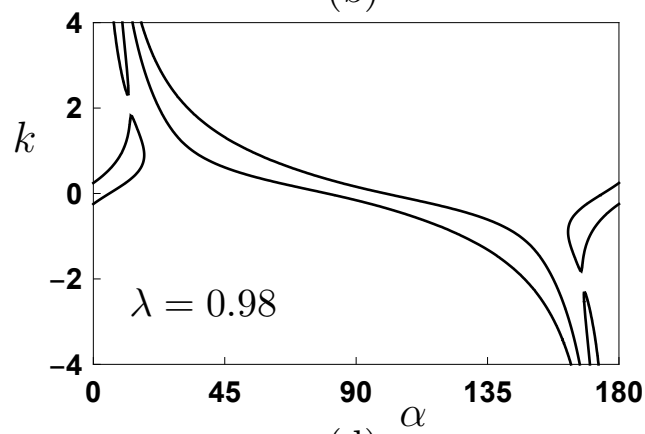

(d)

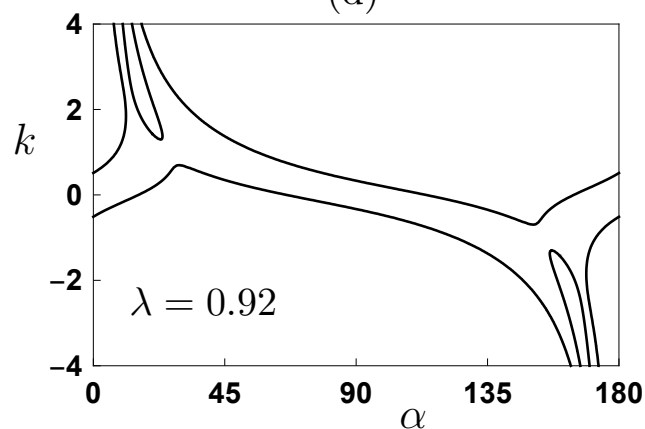

Figure 13: Different $\lambda$ cross sections of the solution of $(25)_{2}$ for $\rho=30$.

Knowles and Sternberg, 1978; Abeyaratne, 1980; Abeyaratne and Knowles, 1989; Merodio and Pence, 2001b). Nevertheless, this requires an intermediate non-elliptic deformation gradient between the two given deformation gradients separated by the strong surface of discontinuity.

Consider the parameter $\bar{k}$ and the deformation gradient $\overline{\mathbf{F}}$ given by (21) on replacing $k$ by $\bar{k}$. The parameter $\bar{k}$ is then understood as the parameter giving the sequence of deformations connecting the deformation in $\Pi^{+}(\bar{k}=0)$ and the deformation in $\Pi^{-}(\bar{k}=k)$. To analyze the ellipticity status of $\overline{\mathbf{F}}$ one has to check (28). If for some pair of orthogonal unit vectors $\mathbf{l}$ and $\mathbf{n}$ such that $\mathbf{l} \cdot \mathbf{n}=0, \overline{\mathbf{F}}$ satisfies the equation $(28)$, then the deformation gradient is said to be non-elliptic. The polynomial expression of $(28)$ for $\overline{\mathbf{F}}$ is a lengthy one and provides no additional insight, and is not therefore included here. We just consider that there exists a set $(\lambda, \bar{k}, \alpha, \rho)$ such that $\overline{\mathbf{F}}$ is non-elliptic, i.e. it satisfies (28). For the purpose of our analysis it is sufficient to show that if $(\lambda, k, \alpha, \rho)$ is a solution of $(25)_{2}$ then there exists a non-elliptic $(\lambda, \bar{k}, \alpha, \rho)$ such that $|\bar{k}| \leq|k|$ (for details, see Merodio, 1999). This guarantees that there exists both a $\bar{k}$ that gives a characteristic direction in the reference configuration that is aligned therein with a shock surface and an intermediate non-elliptic deformation gradient between the two given deformation gradients separated by the strong shock.

For the purpose of this discussion, our attention is now restricted to different fixed $\lambda$ cross sections in order to determine whether or not there exists an intermediate value of $\bar{k}$ such that $\overline{\mathbf{F}}=\mathbf{F}^{-}(\bar{k})$ is non-elliptic. To this end, in Fig. 14 are plotted the solutions $(\lambda, k, \alpha)$ that follow from 25$)_{2}$ (solid curves) and the solutions $(\lambda, \bar{k}, \alpha)$ that follow from (28) (dashed curves) for the reinforcing parameter $\rho=30$ and different fixed $\lambda$ cross sections. It is clearly seen in Fig. 14 that for a mechanically consistent strong shock characterized by $(\lambda, k, \alpha, \rho)$ there always exists at least one value of $\bar{k}$, with $|\bar{k}| \leq|k|$, such that the set $(\lambda, \bar{k}, \alpha, \rho)$ yields an (intermediate) non-elliptic deformation gradient. Thus, a mechanically consistent strong shock involves loss of ordinary ellipticity at some 
deformation on the $\bar{k}$-parametrized path connecting $\mathbf{F}^{+}$and $\mathbf{F}^{-}$.

(a)

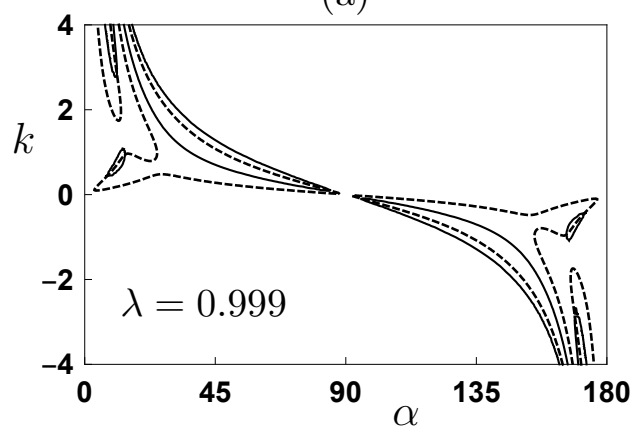

(c)

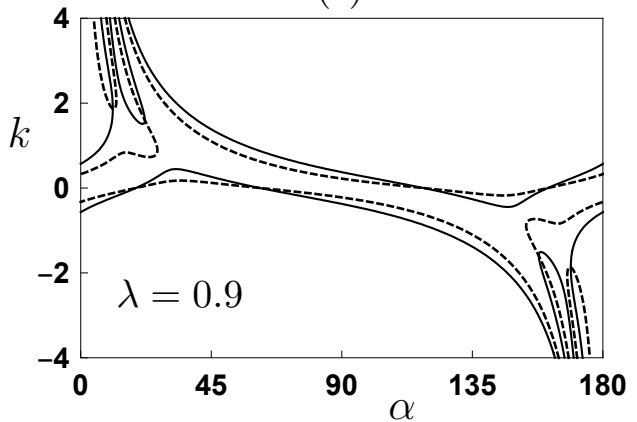

(b)

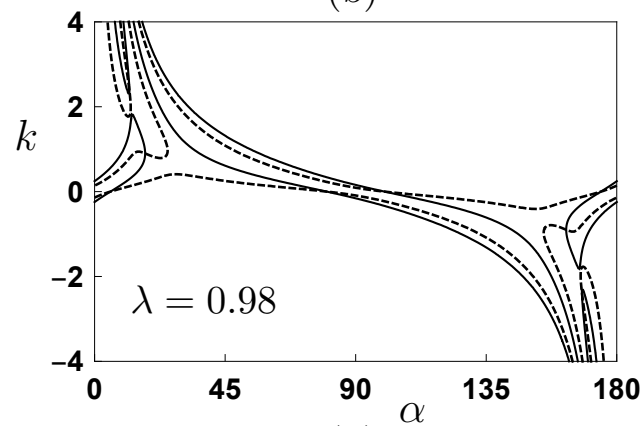

(d)

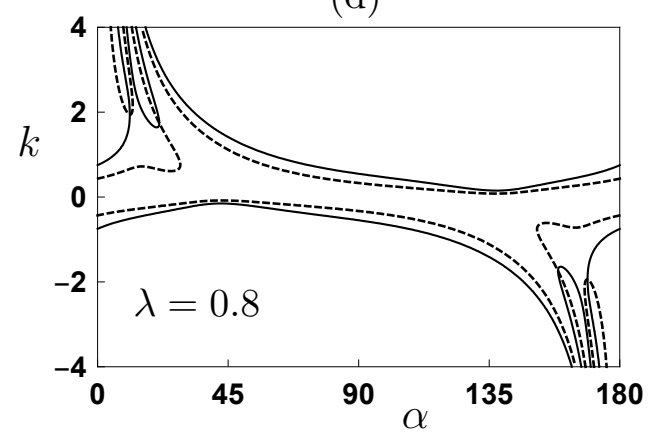

Figure 14: Different $\lambda$ cross sections of $(\lambda, k, \alpha)$ solutions of $(25)_{2}$ (solid curves) and $(\lambda, \bar{k}, \alpha)$ solutions of (28) (dashed curves) for $\rho=30$. Note that for all cross sections both solutions intersect at least when $k=\bar{k}=0$ (weak surfaces). Furthermore, for a given angle $\alpha$ it is easy to check that there exists a $|\bar{k}| \leq|k|$. This establishes that a mechanically strong shock involves loss of ordinary ellipticity at some intermediate deformation gradient on the path connecting $\mathbf{F}^{+}$and $\mathbf{F}^{-}$.

\section{$5 \quad$ Energetically admissible shocks}

\subsection{Shock driving traction}

Various aspects of the theory of finite elastostatics for materials characterized by nonelliptic elastic potentials have been studied in a number of investigations. Under suitable conditions, such materials can sustain equilibrium deformations with jump discontinuities in the derivative of the displacement field and the stress field across the shocks.

A quasi-static motion involving equilibrium elastostatic shocks at each instant may be dissipative. Thus, the existence of an elastostatic shock modifies the mechanical energy balance of the elastic body. For every regular three-dimensional sub-domain $\mathcal{D}$ of the reference configuration, with boundary $\partial \mathcal{D}$, it is required at each instant of time $t$ that

$$
\int_{\partial \mathcal{D}} \mathbf{t} \cdot \mathbf{v} \mathrm{d} A-\frac{\mathrm{d}}{\mathrm{d} t} \int_{\mathcal{D}} W(\mathbf{F}) \mathrm{d} V \geq 0
$$

where $\mathbf{t}$ is the nominal traction vector, $\mathbf{v}$ is the quasi-static particle velocity and $W$ is the elastic energy density per unit reference volume. The first term gives the work done by the external forces acting on $\partial \mathcal{D}$, while the second term represents the elastic energy storage rate. 
At any point of the body that is being traversed by a moving elastostatic shock, the work of the external forces differs from the elastic energy storage rate by the work done in moving the elastostatic shock. The latter can be expressed as the integral over the shock surface, denoted $\mathcal{A}$ at time $t$, of the product of a scalar parameter that represents the socalled shock driving traction, denoted by $\mathcal{S}$, with the normal component of the elastostatic shock velocity, denoted $\mathcal{V}$. It was shown, as in Knowles (1979), that in the presence of a mechanically equilibrated elastostatic shock, the energy balance can be expressed as

$$
\frac{\mathrm{d}}{\mathrm{d} t} \int_{\mathcal{D}} W(\mathbf{F}) \mathrm{d} V=\int_{\partial \mathcal{D}} \mathbf{t} \cdot \mathbf{v} \mathrm{d} A-\int_{\mathcal{A}} \mathcal{S} \mathcal{V} \mathrm{d} A
$$

where $\mathcal{V}$ is the normal velocity component of $\mathcal{A}$ in the reference configuration. The equality (36) holds if and only if the field quantities involved in the considered problem do not have classical smoothness, otherwise the second term on the right-hand side of (36) vanishes and the inequality (35) becomes an equality.

As in Abeyaratne and Knowles (1989), $\mathcal{S}$ is defined as the magnitude of a fictitious nominal traction vector acting on the elastostatic shock by the surrounding material, and Knowles (1979) showed that it is given by

$$
\mathcal{S}=\llbracket W(\mathbf{F})-\mathbf{S N} \cdot \mathbf{F N} \rrbracket_{-}^{+} .
$$

By the traction continuity condition $(10)_{2}$ and the formulas 140 and $(17)$ this can be written

$$
\mathcal{S}=\llbracket W(\mathbf{F}) \rrbracket_{-}^{+}+k(\boldsymbol{\sigma} \mathbf{n}) \cdot \mathbf{l},
$$

where the traction $\boldsymbol{\sigma n}$ can be calculated from either the '+' or '-' side. See also Abeyaratne and Knowles (1989), who showed this result for an incompressible anisotropic nonlinearly elastic material under plane deformation, where $k$ is the strength of the elastostatic shock.

From a variational point of view, Abeyaratne (1983) showed that for the particular case of dead load the criterion of energy stability $\mathcal{S}=0$ is a necessary condition for the displacement field $\mathbf{u}$ to minimize the potential energy over the considered function class. Then, if during the quasi-static motion $\mathcal{S}=0$ at any point of the elastostatic shock, the motion is said to be dissipation free. An equilibrium state for which $\mathcal{S}=0$ is said to satisfy the Maxwell condition, also known as a neutral stability, in which, neither $\Pi^{+}$nor $\Pi^{-}$is energetically favoured.

The Maxwell (line) condition gives rise to non-dissipative solutions in elastostatics and it is just the specialization of the admissibility condition $\mathcal{S}=0$ used for shocks.

An elastostatic shock considered to be advancing into $\Pi^{+}$requires $\mathcal{V}>0$, while the advancement towards $\Pi^{-}$requires $\mathcal{V}<0$. Then, based on the energy balance in (36), the existence of an energetically admissible elastostatic shock requires

- $\mathcal{S} \leq 0(\mathcal{S} \geq 0)$ when the admissible elastostatic shock motion involves $\Pi^{-}\left(\Pi^{+}\right)$ being converted into $\Pi^{+}\left(\Pi^{-}\right)$, i.e. $\Pi^{+}\left(\Pi^{-}\right)$is favoured $\mathcal{V}<0(\mathcal{V}>0)$.

Our attention is naturally restricted to shocks for which $\mathcal{S} \geq 0$. In what follows the focus is on both the neutral stability $\mathcal{S}=0$, which leads to solutions of boundary-value problems, and to maximally dissipative solutions (maxima of $\mathcal{S}$, where $\mathrm{d} \mathcal{S} / \mathrm{d} \alpha=0$ ). Before embarking on the general treatment, let us illustrate this analysis with an enlightening example.

Because of the symmetry noted earlier it is sufficient to consider shocks for which $\alpha \in[0,90]$, and for illustration the solutions of the cross section Fig. 14(b) are shown 
in Fig. 15. The points where $\mathcal{S}=0$ and/or $\mathrm{d} \mathcal{S} / \mathrm{d} \alpha=0$ satisfy $H=0$ are obtained by using (38). In particular, the points 1 and 2 satisfy $\mathcal{S}=0$ (non-dissipative), points 3 and 4 are locations of maximal $\mathcal{S}$, and points 5 and 6 satisfy both conditions. For points 5 and $6, k=0$ the deformation gradient is continuous, and therefore not of interest. The deformation in $\Pi^{-}$, as measured by $C_{11}^{-}$and $C_{12}^{-}$, is obtained for each point using $(23)_{1,2}$, and the results are shown in Table 2 .

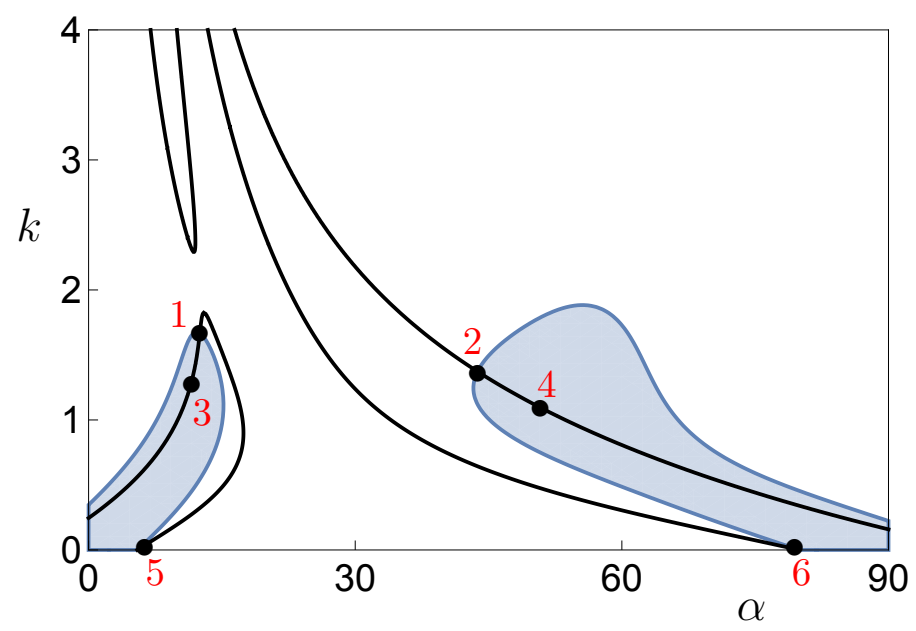

Figure 15: Plot of $H=0$ (continuous curves) and the region $\mathcal{S}>0$ (shaded) showing the locations (bullet points) where $\mathcal{S}=0$ and $\mathrm{d} \mathcal{S} / \mathrm{d} \alpha=0$ for the $\lambda$ cross section in Fig. 14(b) associated with $\lambda=0.98$ and $\rho=30$. The points labelled with the red numbers 1 and 2 satisfy $\mathcal{S}=0$, i.e. are non-dissipative, and points 3 and 4 satisfy $\mathrm{d} \mathcal{S} / \mathrm{d} \alpha=0$. The values $(\alpha, k)$ at these points are approximately 1: $(12.5,1.68) ; 2:(44,1.38) ; 3:(11.6,1.29) ; 4:(50,1.13)$. Also shown are the points 5,6 where $k=0$ and $\alpha=5.5,80$, respectively, with $H=0, \mathcal{S}=0$ and $\mathrm{d} \mathcal{S} / \mathrm{d} \alpha=0$ all satisfied.

\begin{tabular}{lllllll}
\hline \hline Point & 1 & 2 & 3 & 4 & 5 & 6 \\
\hline$C_{11}^{-}$ & 0.41 & 0.52 & 0.53 & 0.61 & 0.96 & 0.96 \\
$C_{12}^{-}$ & 0.93 & -0.9 & 0.86 & -0.83 & 0 & 0 \\
\hline \hline
\end{tabular}

Table 2: Values of $C_{11}^{-}$and $C_{12}^{-}$at the points $1, \ldots, 6$ in Fig. 15.

\subsection{Favourability of $\Pi^{-}$versus $\Pi^{+}$}

In this section we focus on the neutral stability characterized by $\mathcal{S}=0$. The expression for $\mathcal{S}$ is now given explicitly following (38) for the strain-energy function $W$ in (3) subject to the deformations in (13) and (21). This gives

$$
(\boldsymbol{\sigma} \mathbf{n}) \cdot \mathbf{l}=\mu n_{1} n_{2}\left[\lambda^{2}-\lambda^{-2}+4 \rho \lambda^{4}\left(\lambda^{4}-1\right)\right],
$$

which in turn, on use of (38), yields a polynomial of degree eight in $k$ given by

$$
\mathcal{S}=k^{2} \mu \mathcal{S}_{0}=k^{2} \mu \sum_{n=0}^{6} s_{n} k^{n},
$$


where the coefficients $s_{n}$ are given by

$$
\begin{aligned}
s_{0} & =-\frac{1}{2}\left(\lambda^{2} n_{1}^{2}+\lambda^{-2} n_{2}^{2}\right)-\rho\left[2 \lambda^{8} n_{1}^{2}\left(n_{1}^{2}+7 n_{2}^{2}\right)+\lambda^{4}\left(n_{2}^{2}-n_{1}^{2}-8 n_{1}^{2} n_{2}^{2}\right)-\left(n_{1}^{2}-n_{2}^{2}\right)^{2}\right], \\
s_{1} & =-2 \rho n_{1} n_{2}\left[2 \lambda^{8} n_{1}^{2}\left(3 n_{1}^{2}+7 n_{2}^{2}\right)+\lambda^{4}\left(3 n_{2}^{4}-n_{1}^{4}-6 n_{1}^{2} n_{2}^{2}\right)+n_{1}^{2}-n_{2}^{2}\right], \\
s_{2} & =-\frac{1}{2} \rho\left[\left(1-2 n_{1}^{2} n_{2}^{2}\right)\left(1-8 n_{1}^{2} n_{2}^{2}\right)+2 \lambda^{8} n_{1}^{4}\left(3 n_{1}^{4}+30 n_{1}^{2} n_{2}^{2}+35 n_{2}^{4}\right)\right. \\
& \left.+2 \lambda^{4} n_{1}^{2}\left(n_{1}^{2}-n_{2}^{2}\right)\left(n_{1}^{4}-6 n_{1}^{2} n_{2}^{2}-15 n_{2}^{4}\right)\right], \\
s_{3} & =-2 \rho n_{1} n_{2}\left[\left(n_{1}^{2}-n_{2}^{2}\right)\left(4 n_{1}^{2} n_{2}^{2}-1\right)+2 \lambda^{8} n_{1}^{4}\left(3 n_{1}^{2}+7 n_{2}^{2}\right)+2 \lambda^{4} n_{1}^{2} n_{2}^{2}\left(5 n_{2}^{2}-3 n_{1}^{2}\right)\right], \\
s_{4} & =-\rho n_{1}^{2}\left[3 n_{2}^{2}\left(n_{1}^{2}-n_{2}^{2}\right)^{2}+2 \lambda^{8} n_{1}^{4}\left(n_{1}^{2}+7 n_{2}^{2}\right)+\lambda^{4} n_{1}^{2}\left(n_{1}^{4}-8 n_{1}^{2} n_{2}^{2}+15 n_{2}^{4}\right)\right], \\
s_{5} & =-2 \rho n_{1}^{3} n_{2}\left[n_{2}^{2}\left(n_{2}^{2}-n_{1}^{2}\right)+2 \lambda^{8} n_{1}^{4}+\lambda^{4} n_{1}^{2}\right], \\
s_{6} & =-\frac{1}{2} \rho n_{1}^{4}\left(n_{2}^{2}+\lambda^{4} n_{1}^{2}\right)^{2} .
\end{aligned}
$$

Here, after ruling out the trivial solution $k=0$ in 40 , the neutral stability reduces to the solutions of

$$
\mathcal{S}_{0}=\sum_{n=0}^{6} s_{n} k^{n}=0 .
$$

Then, a mechanically consistent shock $(\lambda, k, \alpha, \rho)$ satisfying $(25)_{2}$ is non-dissipative if and only if it is neutrally stable. Therefore, a non-dissipative mechanically consistent elastostatic shock characterized by $(\lambda, k, \alpha, \rho)$ must satisfy the system of equations

$$
H=\mathcal{S}_{0}=0 .
$$

Figs. 16(a) and (b) show, respectively, values of $\alpha$ versus $\lambda$ and values of $k$ versus $\lambda$ obtained from (43) for $\rho=1$. Corresponding results for $\rho=1.3$ are shown in Fig. 17. Let us focus first on $\rho=1$. As $\lambda$ decreases from the undeformed configuration, i.e. as fibre contraction is increased, non-dissipative shocks nucleate where the first mode associated with loss of ellipticity for $\mathbf{F}^{+}$occurs. It follows that, firstly, non-dissipative shocks nucleate when $\lambda=\lambda_{o}$ for which $\alpha=90$, as shown in Fig. 16(a), the curve being associated with fibre kinking. Due to the symmetry this curves gives rise to kink bands associated with a three-zone state $\mathbf{F}^{+} / \mathbf{F}_{\mathrm{K}}^{-} / \mathbf{F}^{+}$, where the index $\mathrm{K}$ refers to the kinking zone.
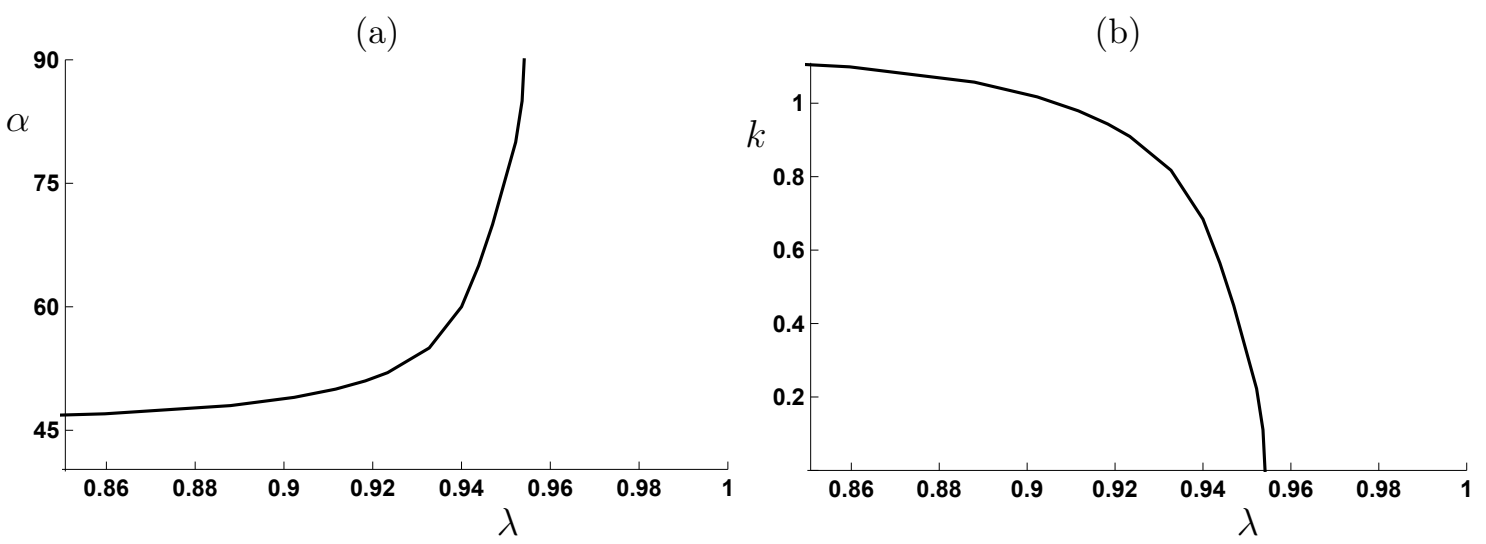

Figure 16: Plot of (a) $\alpha$ versus $\lambda$ and (b) $k$ versus $\lambda$ with $\rho=1$.

It was shown in Section 3.3 that fibre splitting is possible if and only if $\rho>\hat{\rho}^{*}$, where $\hat{\rho}^{*} \approx 1.298$. It follows that $\rho=1.3$ is associated with fibre splitting but the associated values of $\lambda$ are not shown since they are small, as has been described in Section 3.3 . 

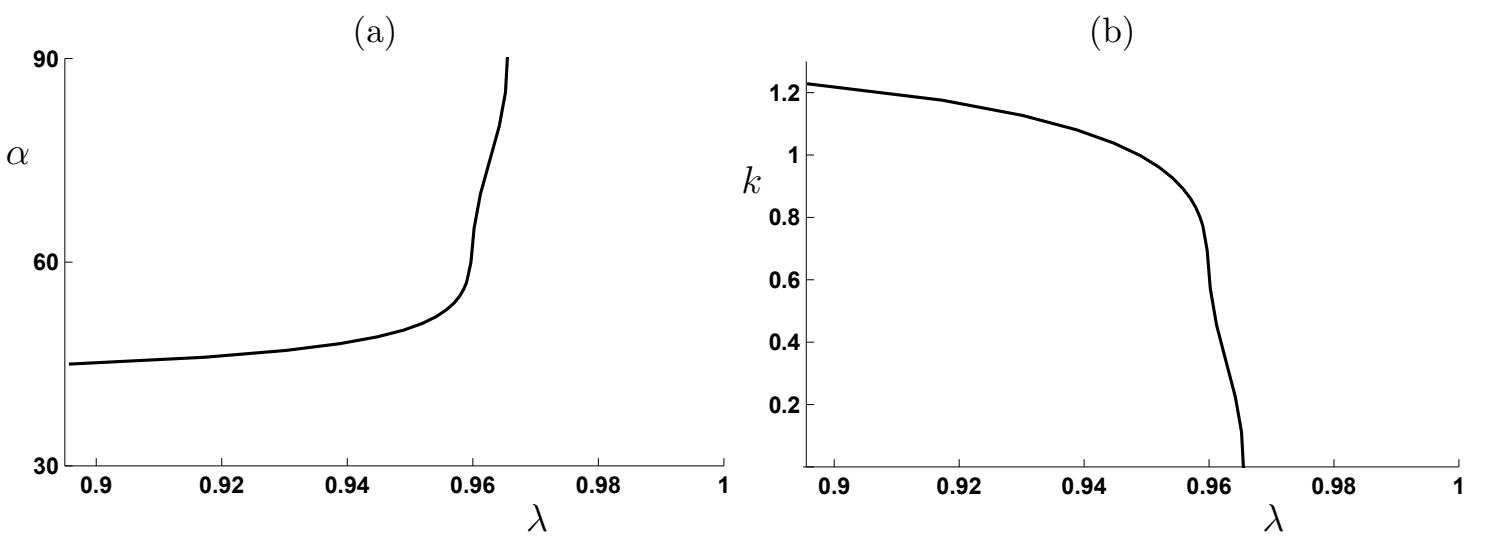

Figure 17: Plot of (a) $\alpha$ versus $\lambda$ and (b) $k$ versus $\lambda$ with $\rho=1.3$.

Nevertheless, as $\lambda$ decreases further a second family of non-dissipative shocks, associated with fibre splitting, nucleates when $\lambda=\lambda_{p}$ for which $\alpha=0$. Similarly to fibre kinking, and again due to the symmetry, fibre splitting also manifests a three-zone state, given by $\mathbf{F}^{+} / \mathbf{F}_{\mathrm{S}}^{-} / \mathbf{F}^{+}$, where the index $\mathrm{S}$ refers to the splitting zone. Therefore, the incipient loss of ellipticity gives rise to non-dissipative shocks, associated with the nucleation of two different modes, one with fibre kinking and other with fibre splitting (for any value of $\rho$, subject to $\rho>\hat{\rho}^{*}$ in the case of splitting). For instance, Figs. 18 and 19 , respectively for $\rho=3$ and $\rho=30$, show values of $\alpha$ versus $\lambda$ and $k$ versus $\lambda$ derived from (43), the continuous curves being associated with fibre kinking and the dashed curves with fibre splitting. For sufficiently large values of $\rho$ it can be seen that kinks can exist even if the condition $0<\lambda<\lambda_{o}$ does not hold. Furthermore, the continuous curves in Figs. 19 include values $\lambda>1$. This means that 43 does not provide for the selection of a unique kink for a given material parameter $\rho$ and load parameter $\lambda$. This is to be expected in view of analogous non-uniqueness results in finite elastic descriptions of a variety of problems involving discontinuous deformation gradients. In this framework it may be concluded that the process is non-dissipative and a more restrictive condition needs to be specified to rule out certain solutions. The formation and growth of such a kink band must be obtained by examining the admissibility and stability of the kinks described and the criterion has to eliminate the snap-back that the non-dissipative condition allows.
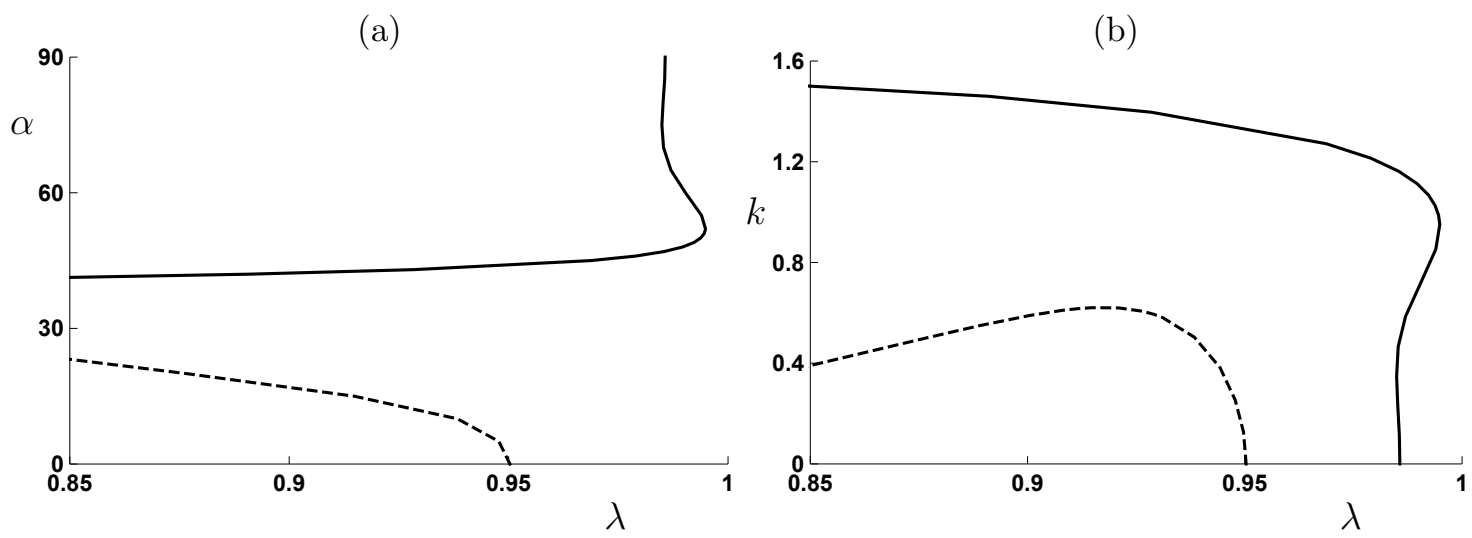

Figure 18: Plot of (a) $\alpha$ versus $\lambda$ and (b) $k$ versus $\lambda$ with $\rho=3$. The dashed curve corresponds to splitting and the continuous curve to kinking.

The deformation in $\Pi^{-}$can be obtained for each non-dissipative shock solution using 
(a)

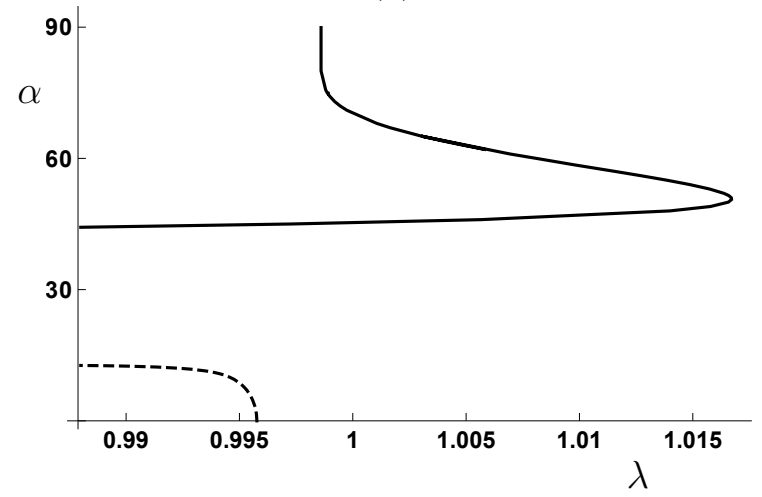

(b)

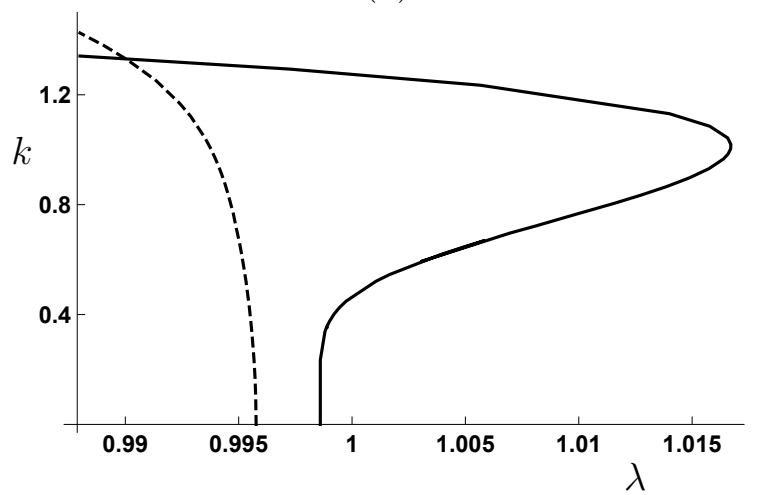

Figure 19: Plot of (a) $\alpha$ versus $\lambda$ and (b) $k$ versus $\lambda$ with $\rho=30$. The dashed curve corresponds to splitting and the continuous curve to kinking.

(23) ${ }_{1,2}$. This yields a better understanding of the fibre behaviour in the zones of localized deformation. For a complete picture and to further illustrate the physical meaning of the solutions shown in Fig. 18 for $\rho=3$ and Fig. 19 for $\rho=30$, using $(23)_{1}$ we plot $C_{11}^{-}$against $\lambda$ for $\rho=3$ and $\rho=30$ in Fig. 20(a) and Fig. 21(a), respectively, and using $(23)_{2}$ we plot $C_{12}^{-}$against $\lambda$ in Fig. 20(b) and Fig. 21(b) for the two values of $\rho$. The evolution of the kinking angle $\phi$ with $\lambda$ for all the non-dissipative shocks is shown in Fig. 22 .

(a)

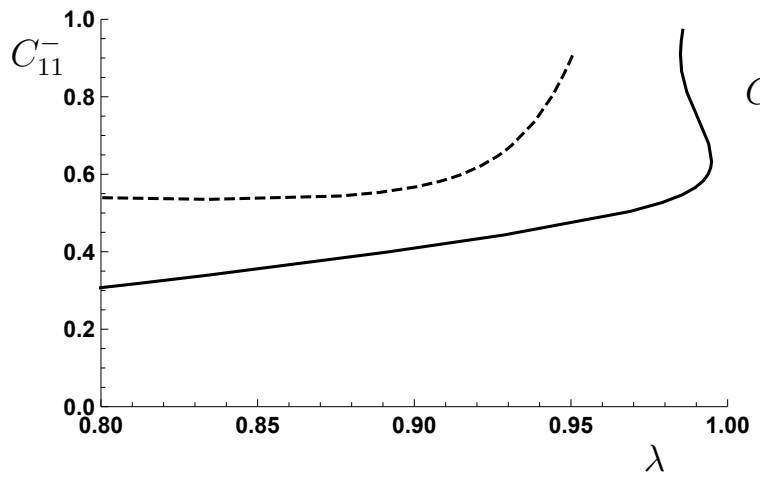

(b)

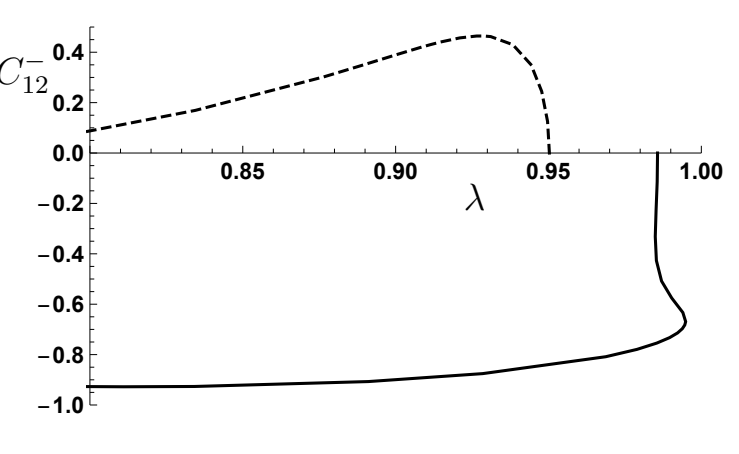

Figure 20: Plot of (a) $C_{11}^{-}$versus $\lambda$ and (b) $C_{12}^{-}$versus $\lambda$ with $\rho=3$. The dashed curve corresponds to splitting and the continuous curve to kinking.

Let us focus on the values of $C_{11}^{-}$and $C_{12}^{-}$associated with the non-dissipative shocks that nucleate with the incipient loss of ellipticity, i.e. we focus on the solutions that are associated with the values $\lambda=\lambda_{o}$ for fibre kinking and $\lambda=\lambda_{p}$ for fibre splitting. Quasi-static evolution of the two families of shocks is described as follows. With respect to fibre kinking (at $\lambda=\lambda_{o}$ ), Figs. 20(a) and 21(a) show that the nucleation of nondissipative motion or neutral stability from the values on the ' + ' side gives rise to $\mathbf{F}_{\mathrm{K}}^{-}$, and involves a simultaneous rapid large increase of the fibre contraction as measured by $C_{11}^{-}$(continuous curves) together with a rapid escalation of fibre shearing $C_{12}^{-}$, which is shown in Figs. 20(b) and 21(b) (continuous curves). This effect is also shown for fibre splitting, i.e. the nucleation of non-dissipative motion from the values on the ' + ' side gives rise to $\mathbf{F}_{\mathrm{S}}^{-}$(dashed curves in each case), and involves a simultaneous rapid large increase of the fibre contraction together with a rapid escalation of fibre shearing. The fibres are highly contracted and undergo large shear deformations. Nevertheless, for sufficiently large 
(a)

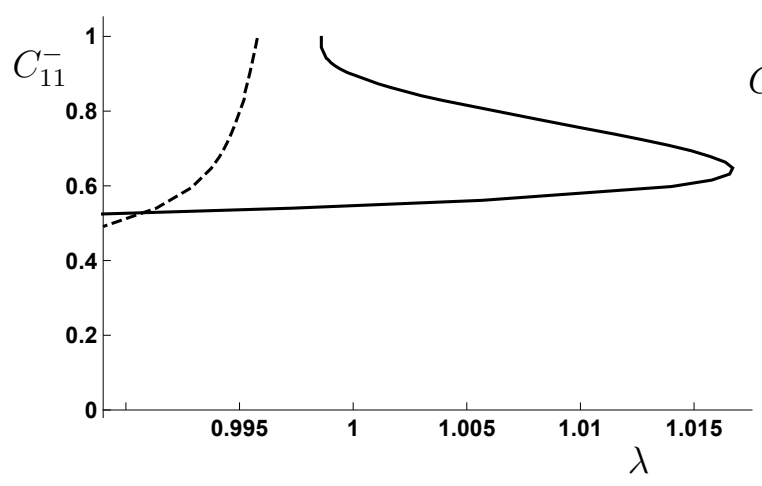

(b)

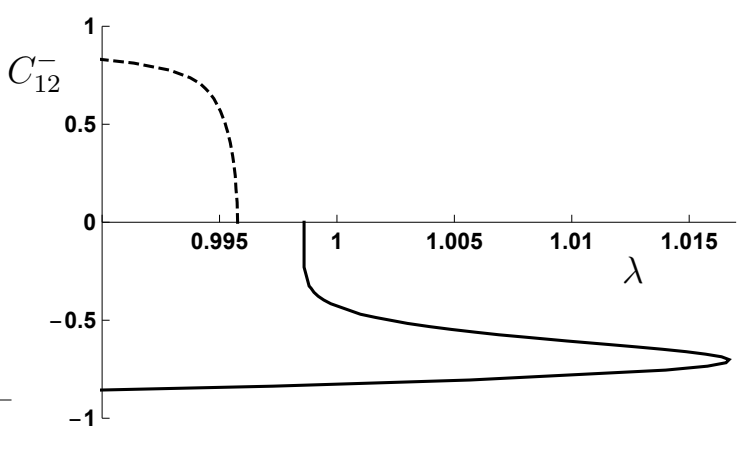

Figure 21: Plot of (a) $C_{11}^{-}$versus $\lambda$ and (b) $C_{12}^{-}$versus $\lambda$ with $\rho=30$. The dashed curve corresponds to splitting and the continuous curve to kinking.

(a)

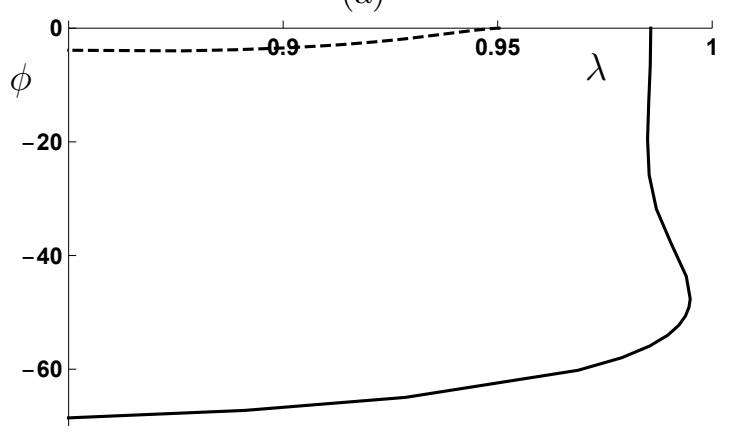

(b)

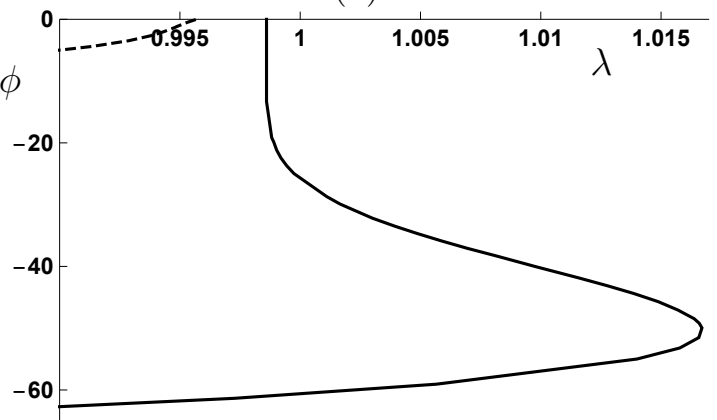

Figure 22: Plot of $\phi$ versus $\lambda$ : (a) $\rho=3$; (b) $\rho=30$. The dashed curve corresponds to splitting and the continuous curve to kinking.

values of $\rho$, for instance $\rho=3$, we find that kinks can exist even for $\lambda>\lambda_{o}$. The process is non-dissipative and a more restrictive condition needs to be specified to rule out these solutions. The formation and growth of such a kink band must be obtained by examining the admissibility and stability of the kinks described and an admissibility criterion has to eliminate the snap-back that the non-dissipative condition allows.

\subsection{Maximally dissipative shocks}

We recall that the shock driving traction $\mathcal{S}$ determines the local rate of mechanical energy dissipation. A mechanically consistent elastostatic shock that creates $\Pi^{-}$at the expense of $\Pi^{+}$requires (necessary but not sufficient) $\mathcal{S} \geq 0$ to be energetically admissible. It was shown in Merodio and Pence (2001b) that, for a given $\lambda$, the shock angle $\alpha$ parametrizes the energetically admissible elastostatic shocks of strength $k$ which in turn determines the dependence of $\mathcal{S}$ on $\alpha$ at fixed $\lambda$ and $\rho$. We now look for the energetically admissible shocks that maximize $\mathcal{S}$; its maximum is denoted $\mathcal{S}_{\max }$. Based on the interpretation of the shock driving traction, these particular shocks correspond to a maximum traction exerted by the surrounding material. Then maximally dissipative elastostatic shocks require that $\mathrm{d} \mathcal{S} / \mathrm{d} \alpha=0$, and when combined with $(25)_{2}$ they are given by

$$
\frac{\mathrm{d} \mathcal{S}}{\mathrm{d} \alpha}=0, \quad H=0, \quad \mathcal{S} \geq 0
$$


The derivative of $\mathcal{S}$ with respect to $\alpha$ is obtained using (40) as

$$
\mu^{-1} \frac{\mathrm{d} \mathcal{S}}{\mathrm{d} \alpha}=k\left[k \frac{\partial S_{0}}{\partial \alpha}+\left(k \frac{\partial S_{0}}{\partial k}+2 S_{0}\right) \frac{\mathrm{d} k}{\mathrm{~d} \alpha}\right]
$$

where the derivative of $k$ with respect to $\alpha$ is obtained using $(25)_{2}$ as

$$
\frac{\mathrm{d} k}{\mathrm{~d} \alpha}=-\frac{\partial H}{\partial \alpha} / \frac{\partial H}{\partial k}
$$

and hence

$$
\mu^{-1} \frac{\mathrm{d} \mathcal{S}}{\mathrm{d} \alpha}=k\left[k \frac{\partial S_{0}}{\partial \alpha}-\left(k \frac{\partial S_{0}}{\partial k}+2 S_{0}\right) \frac{\partial H}{\partial \alpha} / \frac{\partial H}{\partial k}\right] .
$$

Each family of non-dissipative shocks gives rise to a family of maximally dissipative shocks. We focus on the maximally dissipative shocks associated with the non-dissipative shocks that nucleate with the incipient loss of ellipticity.

Figs. 23(a) and (b), respectively, show plots of $\alpha$ and $k$ against $\lambda$ for $(\lambda, k, \alpha)$ satisfying (44) for $\mathcal{S}_{\max }$ with $\rho=3$, and in each case for the fibre kinking $(\mathrm{F}-\mathrm{K})$ and fibre splitting (F-S) failure modes. Parallel results are shown for $\rho=30$ in Figs. 24 (a) and (b). Plots of the angle $\phi$ from (24) are shown in Fig. 25.

(a)

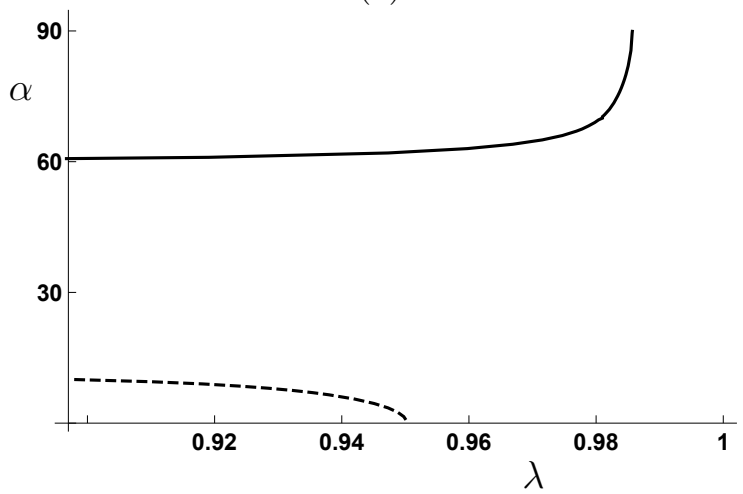

(b)

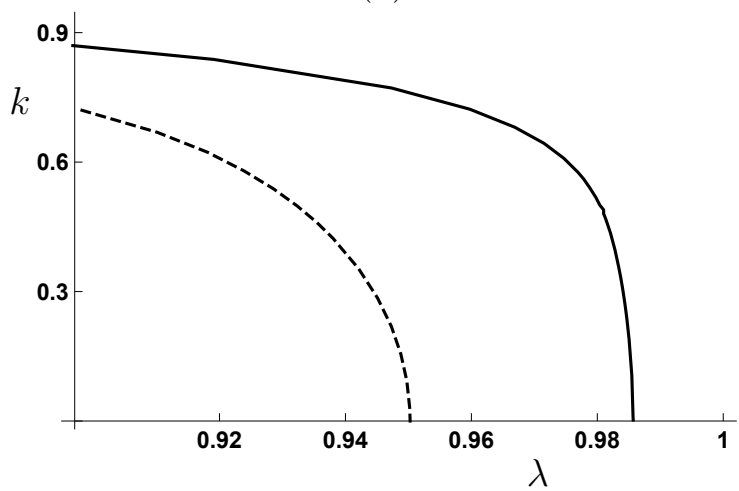

Figure 23: Plot of (a) $\alpha$ versus $\lambda$ and (b) $k$ versus $\lambda$ with $\rho=3$. The dashed curve corresponds to splitting and the continuous curve to kinking.

The criterion of maximum dissipation singles out a unique physically admissible elastostatic shock from the energetically admissible elastostatic shocks, unlike the situation for non-dissipative ones. In addition, the maximally dissipative elastostatic shock is shown to capture closely features observed in different experiments of kink band formation with splitting in fibre-reinforced materials. In particular, it follows from Fig. 25 that $\phi$ at $\mathcal{S}_{\max }$ is almost zero for fibre splitting while the fibre rotation associated with kink formation involves three phases: an initial phase of rapid rotation, a second phase of slow rotation, and a final phase of essentially constant orientation.

Plots of $C_{11}^{-}$and $C_{12}^{-}$versus $\lambda$ for the values $(\lambda, k, \alpha)$ satisfying (44) associated with fibre kinking (continuous curves) and fibre splitting (dashed curves) are shown in Fig. 26 for $\rho=3$ and Fig. 27 for $\rho=30$. The nucleation of maximally-dissipative motion from the values on the ' + ' side (decreasing $\lambda$ ) gives rise to $\mathbf{F}_{\mathrm{S}}^{-}$, which involves a rapid escalation of fibre shearing (as measured by $C_{12}^{-}$). This is also the case for non-dissipative shocks at the incipient loss of ellipticity. Maximally dissipative shocks also show a large 

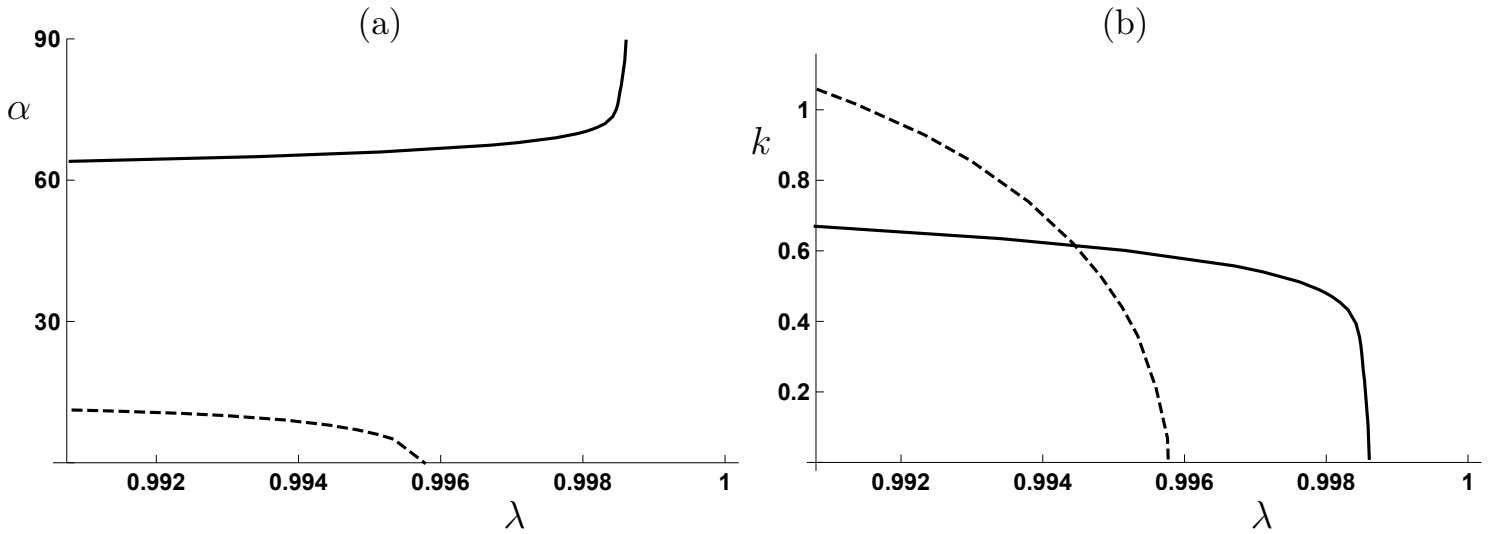

Figure 24: Plot of (a) $\alpha$ versus $\lambda$ and (b) $k$ versus $\lambda$ with $\rho=30$. The dashed curve corresponds to splitting and the continuous curve to kinking.

(a)

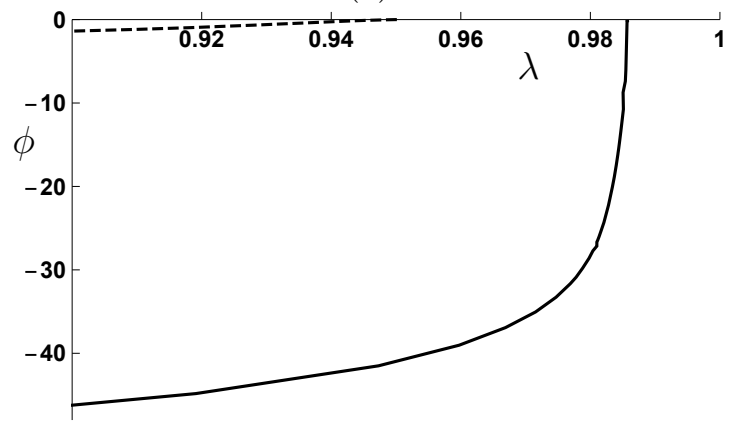

(b)

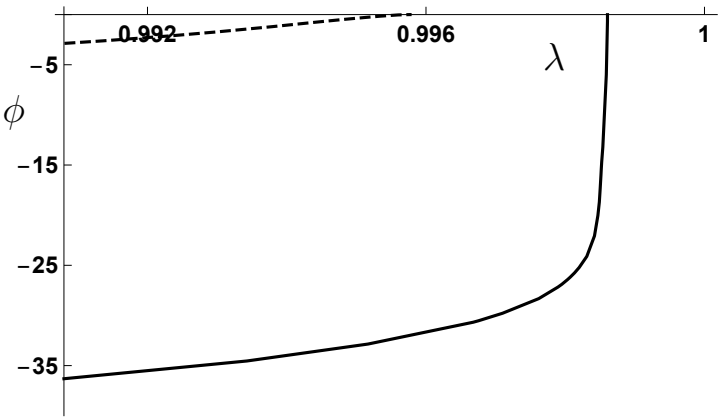

Figure 25: Plot of $\phi$ versus $\lambda$ : (a) $\rho=3$; (b) $\rho=30$. The dashed curve corresponds to splitting and the continuous curve to kinking.

increase of the fibre contraction from the ' + ' side values (as measured by $C_{11}^{-}$) as is also the case for non-dissipation. This can be appreciated by comparing the values of $C_{11}^{-}$and $C_{12}^{-}$in Figs. 26 and 27 with those in Figs. 20 and 21, respectively, for both kinking and splitting. The transverse strain, as measured by $C_{22}^{-}$, as well as $C_{12}^{-}$, has an important role in the fibre splitting as it increases during the compression as $\lambda$ decreases. It is given by the incompressibility condition in the form $C_{22}^{-}=\left[1+\left(C_{12}^{-}\right)^{2}\right] / C_{11}^{-}$, and increases as $C_{12}^{-}$ increases (in magnitude) and $C_{11}^{-}$decreases together.

(a)

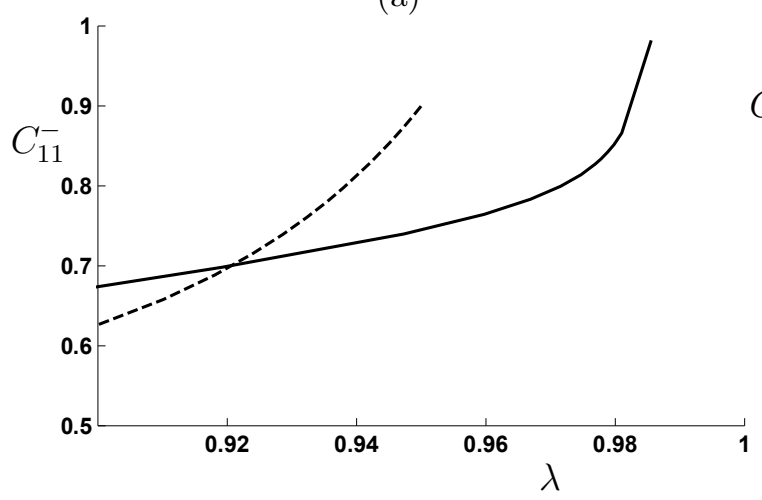

(b)

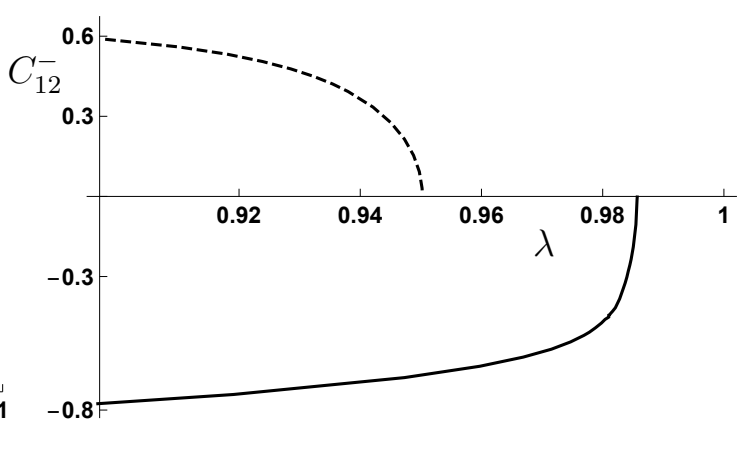

Figure 26: Plot of (a) $C_{11}^{-}$versus $\lambda$ and (b) $C_{12}^{-}$versus $\lambda$ with $\rho=3$. The dashed curve corresponds to splitting and the continuous curve to kinking. 


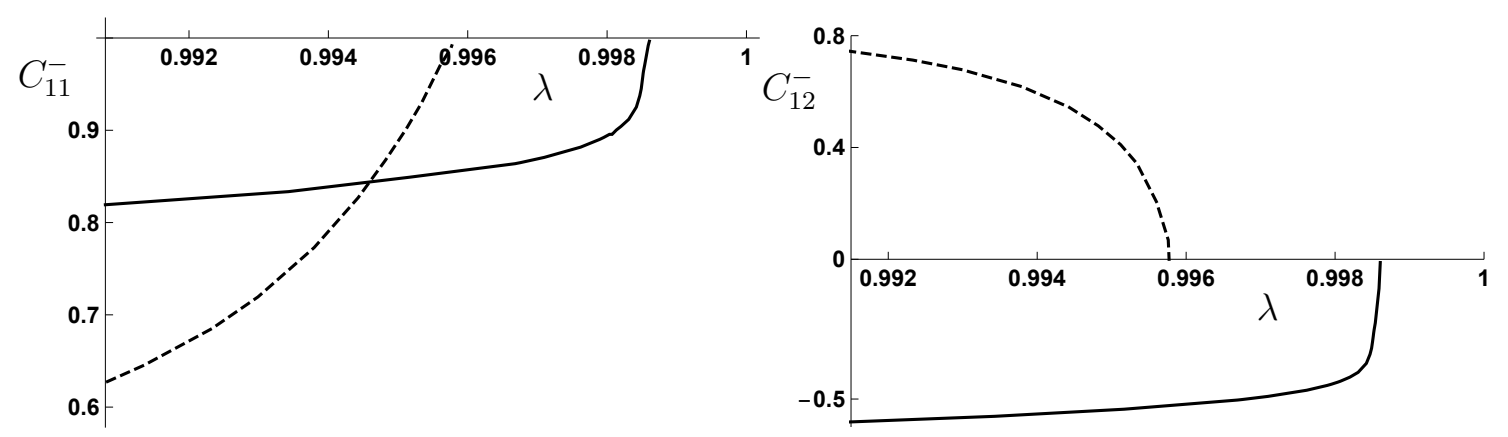

Figure 27: Plot of (a) $C_{11}^{-}$versus $\lambda$ and (b) $C_{12}^{-}$versus $\lambda$ with $\rho=30$. The dashed curve corresponds to splitting and the continuous curve to kinking.

It is straightforward to show, on use of the formula (4) for $I_{5}$, which captures simultaneously fibre stretch and fibre shearing in both $\mathrm{F}-\mathrm{S}$ and $\mathrm{F}-\mathrm{K}$ modes for both $\mathcal{S}_{\max }$ and $\mathcal{S}=0$, that just after shock nucleation the values of $I_{5}^{-}$associated with $\mathcal{S}_{\max }$ are in all cases greater than those of $I_{5}^{+}$at nucleation (given by the loss of ellipticity on the ' + ' side). The maximally dissipative elastostatic shocks are related to a non-elliptic deformation on one half-space and an elliptic deformation on the other half-space. It is therefore tempting to conclude that as elliptic deformations are energetically favoured maximizing the dissipative inequality may be a reasonable condition for singling out a physically and mechanically admissible elastostatic shock.

\section{Conclusions}

It can be argued that fibre splitting is not related to continuous displacements as it is a catastrophic failure mechanism. However, the approach that we have adopted here provides useful data for understanding and describing the failure mechanisms. Furthermore, we have shown that connecting the invariants $I_{4}$ and $I_{5}$ with the formation of shocks, i.e. with the formation of different fibre failure mechanisms, can be exploited in the nonlinear constitutive modelling of fibre reinforced materials.

The shearing indicator $C_{12}$ captured by the invariant $I_{5}$ produces different solutions in comparison with those given by considering the invariant $I_{4}$ alone in the constitutive model (see Merodio and Pence, 2001a b). This implies that shearing between the matrix and fibre in composite (heterogeneous) materials is associated with possible failure by fibre splitting.

The considered failure mechanisms have been shown to be clearly dissipative. Furthermore, they give rise to two-phase piecewise homogeneous plane deformations in which the phase being created suffers a rapid increase of fibre contraction and fibre shearing compared to the original phase. Maximally dissipative solutions have been analyzed in detail and it has been shown that not only is it possible to single out a unique solution but also these solutions are associated with non-elliptic deformations being converted into elliptic deformations. These features are reasonable for singling out admissible solutions.

\section{Acknowledgement}

The authors acknowledge support from the Ministerio de Ciencia, Spain, under the Project number DPI2011-26167. Mustapha El Hamdaoui also thanks the Ministerio de Economía 
y Competitividad, Spain, for funding under the Project number DPI2008-03769 and Grant number BES-2009-027812.

\section{References}

Abeyaratne, R. 1980. Discontinuous deformation gradients in plane finite elastostatics of incompressible materials. J. Elasticity 10, 255-293.

Abeyaratne, R. 1983. An admissibility condition for equilibrium shocks in finite elasticity. J. Elasticity 13, 175-184.

Abeyaratne, R., Knowles, J.K. 1989. Equilibrium shocks in plane deformations of incompressible elastic materials. J. Elasticity 22, 63-80.

Anand, L., Spitzig, W.A. 1980. Initiation of localized shear bands in plane strain. J. Mech. Phys. Solids 28, 113-128.

Baek, S., Pence, T.J. 2010. Emergence and disappearance of load induced fiber kinking surfaces in transversely isotropic hyperelastic materials. Zeits. Ang. Math. Phys. 61, $745-772$.

Budiansky, B., Fleck, N.A. 1993. Compressive failure of fiber composites. J. Mech. Phys. Solids 41, 183-211.

Christensen, R.M., DeTeresa, S.J. 1997. The kink band mechanism for the compressive failure of fiber composite materials. J. Appl. Mech. 64, 1-6.

Christoffersen, J., Jensen, H.M. 1996. Kink band analysis accounting for the microstructure of fiber reinforced materials. Mech. Mater. 24, 305-315.

El Hamdaoui, M., Merodio, J., Ogden, R.W. 2015. Loss of ellipticity in the combined helical, axial and radial elastic deformations of a fiber-reinforced circular cylindrical tube. Int. J. Solids Struct. 63, 99-108.

El Hamdaoui, M., Merodio, J., Ogden, R.W. 2018. Deformation induced loss of ellipticity in an anisotropic circular cylindrical tube. J. Eng. Math. 109, 31-45.

Fu, Y.B., Freidin, A.B. 2004. Characterization and stability of two-phase piecewisehomogeneous deformations. Proc. R. Soc. A 460, 3065-3094.

Fu, Y.B., Zhang, Y.T. 2006. Continuum-mechanical modelling of kink-band formation in fiber-reinforced composites. Int. J. Solids Struct. 43, 3306-3323.

Hadamard, J. 1903. Leçons sur la propagation des ondes et les équations de l'hydrodynamique. Herrmann, Paris.

Hasanyan, A.D., Waas, A.M. 2018. Compressive failure of fiber composites: a homogenized, mesh-independent model. J. Appl. Mech. 85, 091001-15.

Hill, R. 1962. Acceleration waves in solids. J. Mech. Phys. Solids 10, 1-16.

Hill, R., Hutchinson, J.W. 1975. Bifurcation phenomena in the plane tension test. J. Mech. Phys. Solids 23, 239-264. 
Hutchinson, J.W., Tvergaard, V. 1981. Shear band formation in plane strain. Int. J. Solids Struct. 17, 451-470.

Jensen, H.M., Christoffersen, J. 1997. Kink band formation in fiber reinforced materials. J. Mech. Phys. Solids 45, 1121-1136.

Knowles, J.K. 1979. On the dissipation associated with equilibrium shocks in finite elasticity. J. Elasticity 9, 131-158.

Knowles, J.K., Sternberg, E. 1976. On the failure of ellipticity of the equations for finite elastostatic plane strain. Arch. Ration. Mech. Anal. 63, 321-336.

Knowles, J.K., Sternberg, E. 1978. On the failure of ellipticity and the emergence of discontinuous deformation gradients in plane finite elastostatics. J. Elasticity 8, 329-379.

Knowles, J.K., Sternberg, E. 1980. Discontinuous deformation gradients near the tip of a crack in finite anti-plane shear: an example. J. Elasticity 10, 81-110.

Kyriakides, S., Arseculeratne, R., Perry, E.J., Liechti, K.M. 1995. On the compressive failure of fiber reinforced composites. Int. J. Solids Struct. 32, 689-738.

Kyriakides, S., Ruff, A.E. 1997. Aspects of the failure and postfailure of fiber composites in compression. J. Compos. Mater. 31, 1633-1670.

Lee, S.H., Anthony, M.W. 1999. Compressive response and failure of fiber reinforced unidirectional composites. Int. J. Fracture 100, 275-306.

Lee, S.H., Yerramalli, C.S., Waas, A.M. 2000. Compressive splitting response of glass-fiber reinforced unidirectional composites. Compos. Sci.Technol. 60, 2957-2966.

Merodio, J. 1999. Equilibrium shocks in a directionally reinforced neo-Hookean material under plane deformation. PhD Thesis, Michigan State University.

Merodio, J., Ogden, R.W. 2002. Material instabilities in fiber-reinforced nonlinearly elastic solids under plane deformation. Arch. Mech. 54, 525-552.

Merodio, J., Ogden, R.W. 2003. Instabilities and loss of ellipticity in fiber-reinforced compressible non-linearly elastic solids under plane deformation. Int. J. Solids Struct. 40, $4707-4727$.

Merodio, J., Ogden, R.W. 2005a. On tensile instabilities and ellipticity loss in fiberreinforced incompressible non-linearly elastic solids. Mech. Res. Comm. 32, 290-299.

Merodio, J., Ogden, R.W. 2005b. Tensile instabilities and ellipticity in fiber-reinforced compressible non-linearly elastic solids. Int. J. Eng. Sci. 43, 697-706.

Merodio, J., Ogden, R.W. 2005c. Remarks on instabilities and ellipticity for a fiberreinforced compressible nonlinearly elastic solid under plane deformation. Q. Appl. Math. 63, 325-333.

Merodio, J., Neff, P. 2006. A note on tensile instabilities and loss of ellipticity for a fiberreinforced nonlinearly elastic solid. Arch. Mech. 58, 293-303.

Merodio, J., Pence, T.J. 2001a. Kink surfaces in a directionally reinforced neo-Hookean material under plane deformation: I. Mechanical equilibrium. J. Elasticity 62, 119-144. 
Merodio, J., Pence, T.J. 2001b. Kink surfaces in a directionally reinforced neo-Hookean material under plane deformation: II. Kink band stability and maximally dissipative band broadening. J. Elasticity 62, 145-170.

Moran, P.M., Liu, X.H., Shih, C.F. 1995. Kink band formation and band broadening in fiber composites under compressive loading. Acta Metall. 43, 2943-2958.

Moran, P.M., Shih, C.F. 1998. Kink band propagation and broadening in ductile matrix fiber composites: Experiments and analysis. Int. J. Solids Struct. 35, 1709-1722.

Poulsen, J.S., Moran, P.M., Shih, C.F., Byskov, E. 1997. Kink band initiation and band broadening in clear wood under compressive loading. Mech. Mater. 25, 67-77.

Prabhakar, P., Waas, A.M. 2013a. Interaction between kinking and splitting in the compressive failure of unidirectional fiber reinforced laminated composites. Compos. Struct. $98,85-92$.

Prabhakar, P., Waas, A.M. 2013b Micromechanical modeling to determine the compressive strength and failure mode interaction of multidirectional laminates. Composites A: Appl. Sci. Manufact. 50, 11-21.

Rice, J.R. 1973. The initiation and growth of shear bands. In: Palmer, A.C. (Ed.), Plasticity and soil mechanics. Proc. Symp. Role of Plasticity in Soil Mechanics, Cambridge, pp. 263-274. Cambridge University Engineering Department.

Rice, J.R. 1976. The localization of plastic deformation. In: Koiter, W.T. (Ed.), Proc.14th Int. Congr. Theor. Appl. Mech. (ICTAM), Delft. pp. 207-220. North-Holland, Amsterdam.

Rudnicki, J.W., Rice, J.R. 1975. Conditions for the localization of deformation in pressuresensitive dilatant materials. J. Mech. Phys. Solids 23, 371-394.

Sutcliffe, M.P.F., Fleck, N.A. 1994. Microbuckle propagation in carbon fiber-epoxy composites. Acta Metall. 42, 2219-2231.

Vogler, T.J., Kyriakides, S. 1997. Initiation and axial propagation of kink bands in fiber composites. Acta Materialia 45, 2443-2454.

Yerramalli, C.S., Waas, A.M. 2004. A nondimensional number to classify composite compressive failure. J. Appl. Mech. 71, 402-408. 